HD-THEP-96-06

DAMTP-96-51

\title{
SOME TESTS FOR THE HELICITY STRUCTURE OF THE POMERON IN ep COLLISIONS
}

\author{
by \\ T. Arens, O. Nachtmann \\ Institut für Theoretische Physik \\ Universität Heidelberg \\ Philosophenweg 16, D-69120 Heidelberg, FRG \\ and \\ M. Diehl, P. V. Landshoff \\ Department of Applied Mathematics and Theoretical Physics \\ University of Cambridge \\ Cambridge CB3 9EW, England
}

\begin{abstract}
We discuss the reaction $e p \rightarrow e \tilde{p} X$ in the one-photon exchange approximation, where it is in essence the reaction $\gamma^{*} p \rightarrow \tilde{p} X$. A large rapidity gap is required between the particle or particles of the proton remnant $\tilde{p}$ and those of $X$. We define a suitable azimuthal angle $\varphi$ between a leptonic and a hadronic plane. The dependence of the cross section on $\varphi$ is given explicitly and can be used to extract cross sections and interference terms for the reaction $\gamma^{*} p \rightarrow \tilde{p} X$ corresponding to the various helicities of the virtual photon $\gamma^{*}$. The interference terms can be used to test models for the large rapidity gap events in a sensitive way. We discuss in detail models with factorizing Pomeron exchange and in particular the Donnachie-Landshoff Pomeron model. We make some remarks on soft colour exchange models and on possible effects of QCD background vacuum fields. We conclude with a suggestion to look for Odderon exchange in exclusive deep inelastic high energy reactions like $\gamma^{*} p \rightarrow \tilde{p} \pi^{0}$ and $\gamma^{*} p \rightarrow \tilde{p} \eta$
\end{abstract}




\section{Introduction}

The idea to look for signals of "hard" diffractive reactions in hadron-hadron and lepton-hadron scattering proved to be a very fruitful one [1]-[0]. This and the discovery of "rapidity gap events" at HERA [6], [7] have triggered intensive experimental and theoretical research in "Pomeron", i.e. diffractive physics. Topical questions which are asked today are for instance the following: Is there a "soft" and a "hard" Pomeron? How are these concepts related? Is the "Pomeron" related to an ordinary Regge trajectory (cf. e.g. [8]), to an effective $C=+1$ "photon" exchange [9], to two-gluon exchange [10, to a perturbative gluon "ladder" [11] or to nonperturbative QCD effects [12]-[17]?

In this article we will propose measurements of some angular correlations in diffractive interactions initiated by a virtual photon which may shed some light on the above questions. We will also propose to look for signals of an "Odderon", the $C=-1$ partner of the "Pomeron", in these reactions. To be specific: We consider electron or positron-proton scattering (Fig. 1):

$$
e^{\mp}(k)+p(p) \rightarrow e^{\mp}\left(k^{\prime}\right)+\tilde{p}(\tilde{p})+X\left(p_{X}\right),
$$

where the 4-momenta are indicated in brackets. A large rapidity gap between the group of hadrons $X$ and those of $\tilde{p}$ is required. Further details on our definition of a rapidity gap are given below. The "proton remnant" $\tilde{p}$ can again be a proton or a diffractively excited proton. The kinematics of deep inelastic ep scattering is well known (cf. e.g. [18]). In our paper we want to draw attention to the kinematic similarity of reaction (1.1) with one pion electroproduction:

$$
e^{-}(k)+p(p) \rightarrow e^{-}\left(k^{\prime}\right)+p(\tilde{p})+\pi^{0}\left(p_{\pi}\right) .
$$

The detailed kinematic analysis of (1.2) was done a long time ago [19]-21]. It can in essence be carried over to (1.1) and adapted to the HERA reference system. This we do in sect. 2 of our paper. We consider, as usual, the reaction (1.1) in the one-photon exchange approximation, where we have a quasi two-body process

$$
\begin{aligned}
& \gamma^{*}(q)+p(p) \rightarrow \tilde{p}(\tilde{p})+X\left(p_{X}\right) \\
& \left(q=k-k^{\prime}\right) .
\end{aligned}
$$

The contribution from $Z$-exchange could easily be incorporated. However, for the range of $Q^{2}=-q^{2}$ below $100 \mathrm{GeV}^{2}$ where rapidity gap events have been studied at HERA so far, $Z$-exchange is unimportant, and thus we will neglect it in the following.

As we will see, there is an interesting azimuthal angle $\varphi$ between suitably defined lepton and hadron planes. The dependence of the cross section for reaction (1.1) on this angle can be used to disentangle the cross sections and interference terms for 
the reaction (1.3) corresponding to the various helicities of the virtual photon $\gamma^{*}$. This will be explained in detail in sect. 3. where the main formula is (3.34). This formula makes the dependence of the cross section on $\varphi$ completely explicit, with terms proportional to $1, \cos \varphi, \sin \varphi$ and $\cos 2 \varphi$, where the coefficients depend only on the other kinematic variables. In sect. 4 we will have a look at reaction (1.3) in the rest system of the group $X$ of final-state hadrons. We will show that nonzero values for certain interference terms can only occur if the "Pomeron" carries nonzero helicity. We will then make some remarks on models for rapidity gap events in connection with such interference terms in sect. 5. In sect. 6 we will discuss possible "Odderon" contributions in inelastic electron-proton scattering. Sect. 7 contains our conclusions. In appendix A we discuss some kinematical questions. In appendix B we treat in detail some issues for the case where $\tilde{p}$ in (1.1), (1.3) is a diffractively excited proton. In appendix $\mathrm{C}$ we show how a measurement of the interference terms through the $\varphi$-dependence of the cross section as discussed in sect. 3 can be used to obtain bounds on the cross section for longitudinally polarized photons in (1.3). This could be quite useful since a direct measurement of the longitudinal cross section - or rather a disentangling of the longitudinal and transverse cross sections - is notoriously difficult.

\section{Production of rapidity gap events as quasi two- body $\gamma^{*}-p$ scattering reactions}

We will assume that the internal structure of the final state hadronic systems $X$ and $\tilde{p}$ in (1.1) is not resolved and that only the 4 -momenta $p_{X}$ and $\tilde{p}$ are considered as observables. We have, however, to place some requirements on the criteria used for defining $X$ and $\tilde{p}$ as specified below. For a given c.m. energy $\sqrt{s}$ the final state of (1.1) is then determined completely by 7 variables, for which we can e.g. take the 3 -momenta $\mathbf{k}^{\prime}, \tilde{\mathbf{p}}$ in a suitable system and $\tilde{m}^{2}=\tilde{p}^{2}$, the invariant mass squared of the proton remnant. We collect the definitions of our kinematic variables and some useful formulae for them in tables 1,2 . We always neglect the electron mass but keep the proton mass.

Let us now consider the reaction (1.1) in the rest frame of the original proton. We choose a convenient coordinate system there: The 3-axis in the direction of the $\gamma^{*}$ momentum $\mathbf{q}$, the 1,2 axes such that $\mathbf{p}_{X}$ is in the 1-3 plane (Fig. 2). In this system we define the angle $\varphi$ as the azimuthal angle of the 3-momentum vector of the incoming electron or positron. We have then:

$$
\sin \varphi=\left.\frac{\mathbf{q} \cdot\left[\left(\mathbf{q} \times \mathbf{p}_{X}\right) \times(\mathbf{q} \times \mathbf{k})\right]}{|\mathbf{q}|\left|\mathbf{q} \times \mathbf{p}_{X}\right| \mathbf{q} \times \mathbf{k} \mid}\right|_{R},
$$




$$
\begin{aligned}
& \cos \varphi=\left.\frac{\left(\mathbf{q} \times \mathbf{p}_{X}\right) \cdot(\mathbf{q} \times \mathbf{k})}{\left|\mathbf{q} \times \mathbf{p}_{X}\right||\mathbf{q} \times \mathbf{k}|}\right|_{R} \\
& 0 \leq \varphi<2 \pi
\end{aligned}
$$

Here and in the following the index $R$ indicates quantities referring to the proton rest system.

The definition of $\varphi$ given in (2.1) is the exact analogue of the definition of the corresponding angle for reaction (1.2) used in [19]-21]. For the case of one hadron semi-inclusive electroproduction and jet production the analogous angle is well known [22]. It has been used by experimentalists in fixed target experiments (cf. e.g. 23]) and in the context of HERA physics in a first analysis of H1 data in 24].

One of the main points we want to make is now the following. If the systems $X$ and $\tilde{p}$ in (1.1) are defined by criteria involving only hadronic variables, no leptonic ones, then the dependence of the cross section for reaction $(1.1)$ on $\varphi(2.1)$ can be made completely explicit and can be used to measure cross sections and interference terms for definite helicities of the virtual photon $\gamma^{*}$. Examples for "good" criteria to define $X$ and $\tilde{p}$ are as follows:

Criterion I: We require $\tilde{p}$ to be a single proton and ask it to carry most of the energy of the original proton in the HERA system. To be precise, we require

$$
\xi \leq \xi_{0} \ll 1
$$

where $\xi$ is defined in table 1 and $\xi_{0}$ is some fixed number. Kinematics guarantees then a large rapidity gap between $\tilde{p}$ and all the particles of $X$.

Criterion II: We require $\tilde{p}$ to be a single proton. For $X$ we take the set of all final states with arbitrary number $n$ of particles of momenta $p_{i}(i=1, \ldots, n)$ such that

$$
\begin{aligned}
& \sum_{i=1}^{n} p_{i}=p_{X} \\
& p_{X} \cdot p_{i} \leq M_{\text {cut }}^{2} \ll p_{X} \cdot \tilde{p} \quad \text { for all } i
\end{aligned}
$$

where $M_{\text {cut }}$ is some fixed mass value. This means that in the rest system of $X$ all energies of the particles composing $X$ are limited to $M_{\text {cut }}^{2} / m_{X}$, which is a rotationally invariant condition. Clearly, (2.3) is a rapidity gap condition, since it requires no particles in the phase space between the particles of $X$ and $\tilde{p}$ (Fig. 3).

Criterion III: We allow the system $\tilde{p}$ to consist of a single proton or of a group of particles, the debris of a diffractively excited proton. We require the invariant mass $\tilde{m}\left(\tilde{m}^{2}=\tilde{p}^{2}\right)$ to be limited by some fixed value $\tilde{m}_{c u t}$ :

$$
\tilde{m} \leq \tilde{m}_{c u t}
$$


Furthermore we require as in I:

$$
\xi \leq \xi_{0} \ll 1 .
$$

Criterion IV: For the system $X$ we choose the same requirements as in Criterion II. But instead of requiring $\tilde{p}$ to be a single proton we allow it to consist of a group of $\tilde{n}=1,2, \ldots$ particles with momenta $\tilde{p}_{j}(j=1, \ldots, \tilde{n})$ such that

$$
\begin{aligned}
& \sum_{j=1}^{\tilde{n}} \tilde{p}_{j}=\tilde{p}, \\
& \tilde{p} \cdot \tilde{p}_{j} \leq \tilde{M}_{\text {cut }}^{2} \text { for all } j .
\end{aligned}
$$

Here $\tilde{M}_{c u t}$ is a fixed constant. Typically one would choose $\tilde{M}_{c u t}$ to be of order a few $\mathrm{GeV}$ in order to guarantee that one is in the diffractive region.

The criteria I - IV above define $X$ and $\tilde{p}$ by use of invariants formed from hadron 4-momenta only.

With such criteria to define the systems of final states $X$ and $\tilde{p}$, these can be treated like quasi-particles. In particular, the leptonic vector $\mathbf{k}$ in Fig. 2 can rotate around the axis $\mathbf{q}$ without affecting $X$ and $\tilde{p}$. The hadronic and the leptonic variables, especially the azimuthal angle $\varphi$, are in a sense decoupled. As we will see, this is appropriate from a physics point of view, if we want to study the dynamics of the $\gamma^{*} p$ reaction (1.3). The criteria I and III are, presumably, rather straightforward to implement experimentally. The criteria II and IV require $X$ and $\tilde{p}$ to correspond to rotationally invariant sets of final states in their respective rest systems, but we will not really use this in the present paper. An important property guaranteed by all four criteria is that in the rest frame of $X$ the set of final states is invariant under rotations around the photon axis. This will be essential for our discussion in sect. 4 .

In the remainder of this section we will relate the angle $\varphi(2.1)$ to quantities directly measurable in the HERA system where the 4-momenta of the original proton and electron are given by

$$
p_{H}=\left(\begin{array}{cc}
E_{p, H} & \\
\sqrt{E_{p, H}^{2}-m_{p}^{2}} & \hat{\mathbf{p}}_{H}
\end{array}\right), \quad k_{H}=\left(\begin{array}{cc}
E_{e, H} \\
-E_{e, H} & \hat{\mathbf{p}}_{H}
\end{array}\right), \quad \hat{\mathbf{p}}_{H}=\left(\begin{array}{l}
0 \\
0 \\
1
\end{array}\right) .
$$

Here and in the following quantities referring to the HERA system carry an index $H$.

Let us define two 4 -vectors $l, n$ :

$$
\begin{aligned}
l^{\mu}: & =\frac{1}{m_{p}} \varepsilon^{\mu \nu \rho \sigma} p_{\nu} q_{\rho} k_{\sigma}, \\
n^{\mu}: & =\frac{1}{m_{p}} \varepsilon^{\mu \nu \rho \sigma} p_{\nu} q_{\rho} p_{X \sigma} .
\end{aligned}
$$


We have in the rest system of the original proton:

$$
\begin{aligned}
l_{R} & =\left(\begin{array}{c}
0 \\
\mathbf{q} \times \mathbf{k}
\end{array}\right)_{R}, \\
n_{R} & =\left(\begin{array}{c}
0 \\
\mathbf{q} \times \mathbf{p}_{X}
\end{array}\right)_{R} .
\end{aligned}
$$

From (2.1) and (2.9) we obtain easily

$$
\begin{aligned}
\sin \varphi & =\frac{\sqrt{\nu^{2}+Q^{2}} \varepsilon_{\alpha \beta \gamma \delta} k^{\alpha} p^{\beta} q^{\gamma} \tilde{p}^{\delta}}{m_{p} \sqrt{-l^{2}} \sqrt{-n^{2}}} \\
\cos \varphi & =-\frac{l \cdot n}{\sqrt{-l^{2}} \sqrt{-n^{2}}}
\end{aligned}
$$

These are covariant expressions for $\sin \varphi, \cos \varphi$. To evaluate them in the HERA system we define an auxiliary vector $\mathbf{a}_{\perp}$ by

$$
\begin{aligned}
\left.\mathbf{a}_{\perp}\right|_{H}:= & \left\{y\left(1+\frac{2 x m_{p}^{2}}{s-m_{p}^{2}}\right) \tilde{\mathbf{p}}_{\perp}\right. \\
& \left.-\frac{1}{s-m_{p}^{2}}\left[-t-m_{p}^{2}+\tilde{m}^{2}+2 x y m_{p}^{2}+m_{p}^{2} \frac{4 p_{X} \cdot k}{s-m_{p}^{2}}\right] \mathbf{q}_{\perp}\right\}_{H}
\end{aligned}
$$

In the HERA system we have for the transverse momenta

$$
\begin{gathered}
\left(\mathbf{q}_{\perp}+\mathbf{k}_{\perp}^{\prime}\right)_{H}=0, \\
\left(\mathbf{q}_{\perp}-\tilde{\mathbf{p}}_{\perp}-\mathbf{p}_{X \perp}\right)_{H}=0, \\
\left.\mathbf{q}_{\perp}^{2}\right|_{H}=Q^{2}\left[1-y-\frac{m_{p}^{2} Q^{2}}{\left(s-m_{p}^{2}\right)^{2}}\right] \\
=x y\left(s-m_{p}^{2}\right)\left[1-y-\frac{x y m_{p}^{2}}{s-m_{p}^{2}}\right] .
\end{gathered}
$$

This leads us to

$$
\begin{aligned}
l_{H} & =-\frac{s-m_{p}^{2}}{2 m_{p}}\left(\begin{array}{c}
0 \\
\mathbf{q}_{\perp} \times \hat{\mathbf{p}}
\end{array}\right)_{H}, \\
n_{H} & =-\left(\begin{array}{l}
\frac{1}{m_{p}}\left(\mathbf{q}_{\perp} \times \tilde{\mathbf{p}}_{\perp}\right) \cdot \mathbf{p} \\
\frac{s-m_{p}^{2}}{2 m_{p}} \mathbf{a}_{\perp} \times \hat{\mathbf{p}}+\frac{p^{0}}{m_{p}} \mathbf{q}_{\perp} \times \tilde{\mathbf{p}}_{\perp}
\end{array}\right)_{H},
\end{aligned}
$$




$$
\begin{aligned}
-l^{2}= & \left.\left(\frac{s-m_{p}^{2}}{2 m_{p}}\right)^{2} \mathbf{q}_{\perp}^{2}\right|_{H}, \\
-n^{2}= & \left\{\left(\frac{s-m_{p}^{2}}{2 m_{p}}\right)^{2} \mathbf{a}_{\perp}^{2}+\left(\mathbf{q}_{\perp} \times \tilde{\mathbf{p}}_{\perp}\right)^{2}\right\}_{H} \\
= & \left(\frac{s-m_{p}^{2}}{2 m_{p}}\right)^{2}\left\{y^{2}\left[-t(1-x)+m_{p}^{2} x(1-x)-\tilde{m}^{2} x\right]\right. \\
& +y\left(s-m_{p}^{2}\right)^{-1}\left[\left(t+m_{p}^{2}-\tilde{m}^{2}\right)\left(m_{X}^{2}-t\right)+x\left(t+m_{p}^{2}-\tilde{m}^{2}\right)^{2}-2 m_{p}^{2} t\right. \\
& \left.\left.\left.-2 m_{p}^{2} m_{X}^{2}\right)\right]-\left(s-m_{p}^{2}\right)^{-2} m_{p}^{2}\left(m_{X}^{2}-t\right)^{2}\right\} \\
& \sin \varphi=y^{1 / 2}\left(y+\frac{4 x m_{p}^{2}}{s-m_{p}^{2}}\right)^{1 / 2}\left(\mathbf{q}_{\perp} \times \tilde{\mathbf{p}}_{\perp}\right) \cdot \hat{\mathbf{p}} \\
& \left.\cdot\left|\mathbf{q}_{\perp}\right|^{-1}\left[\mathbf{a}_{\perp}^{2}+\frac{4 m_{p}^{2}\left(\mathbf{q}_{\perp} \times \tilde{\mathbf{p}}_{\perp}\right)^{2}}{\left(s-m_{p}^{2}\right)^{2}}\right]_{H}^{-1 / 2}\right|_{H} \\
& \cos \varphi=\left.\left(\mathbf{q}_{\perp} \cdot \mathbf{a}_{\perp}\right)\left|\mathbf{q}_{\perp}\right|^{-1}\left[\mathbf{a}_{\perp}^{2}+\frac{4 m_{p}^{2}\left(\mathbf{q}_{\perp} \times \tilde{\mathbf{p}}_{\perp}\right)^{2}}{\left(s-m_{p}^{2}\right)^{2}}\right]^{-1 / 2}\right|_{H}
\end{aligned}
$$

These are the desired expressions for $\sin \varphi, \cos \varphi$ in terms of quantities directly measurable in the HERA system. At HERA we have $s \simeq 9 \cdot 10^{4} \mathrm{GeV}^{2}$ and thus in the kinematic region where rapidity gap events are studied today [6], [7]:

$$
s-m_{p}^{2} \gg m_{p}^{2}, \tilde{m}^{2}, m_{X}^{2}, Q^{2},(-t) .
$$

In this limit, i.e. for $s \rightarrow \infty$, we get from (2.11), (2.16):

$$
\begin{aligned}
& \left.\left.\mathbf{a}_{\perp}\right|_{H} \rightarrow y \tilde{\mathbf{p}}_{\perp}\right|_{H}, \\
& \sin \varphi \rightarrow \sin \varphi^{\prime}=\left.\frac{\left(\mathbf{q}_{\perp} \times \tilde{\mathbf{p}}_{\perp}\right) \cdot \hat{\mathbf{p}}}{\left|\mathbf{q}_{\perp}\right|\left|\tilde{\mathbf{p}}_{\perp}\right|}\right|_{H}, \\
& \cos \varphi \rightarrow \cos \varphi^{\prime}=\left.\frac{\mathbf{q}_{\perp} \cdot \tilde{\mathbf{p}}_{\perp}}{\left|\mathbf{q}_{\perp}\right|\left|\tilde{\mathbf{p}}_{\perp}\right|}\right|_{H} .
\end{aligned}
$$

The angle $\varphi^{\prime}$ is the azimuthal angle between $\mathbf{q}_{\perp}$ and $\tilde{\mathbf{p}}_{\perp}$ in the HERA system. This is illustrated in Fig. 4. Let $\tilde{\psi}$ and $\psi^{\prime}$ be the azimuthal angles of $\tilde{\mathbf{p}}_{\perp}$ and $\mathbf{k}_{\perp}^{\prime}$ with respect to some fixed axes 1,2 in the HERA system, where the 3-axis is defined by the momentum vector of the incoming proton. We have then for large $s$ (cf. (2.17)):

$$
\varphi \approx \varphi^{\prime}=\tilde{\psi}-\psi^{\prime}+\pi
$$

To assess how good this approximation is we observe that at finite $s$ the replacement (2.18) becomes bad for very small $\left|\tilde{\mathbf{p}}_{\perp}\right|_{H}$. Indeed from (2.11) we see that this happens 
for

$$
\left|\tilde{\mathbf{p}}_{\perp}\right|_{H} \lesssim \frac{\left|\mathbf{q}_{\perp}\right|_{H}}{y\left(s-m_{p}^{2}\right)} \cdot\left(-t-m_{p}^{2}+\tilde{m}^{2}\right) .
$$

Now we have $\left|\tilde{\mathbf{p}}_{\perp}\right|_{H}^{2} \approx|t|$ with corrections of order $x|t|$ and $x^{2} m_{p}^{2}$, and $\left|\mathbf{q}_{\perp}\right|_{H}^{2}=$ $Q^{2}(1-y) \cdot\left(1+O\left(x m_{p}^{2} / s\right)\right)(\mathrm{cf}$. $(2.13))$. From this we find that the relative error which is made by using $\sin \varphi^{\prime}$ instead of $\sin \varphi$ is of order

$$
O\left(\frac{\left|\tilde{\mathbf{p}}_{\perp}\right|_{H}}{Q} \cdot \frac{m_{p}^{2}, \tilde{m}^{2},|t|}{y s}\right)=O\left(x \cdot \frac{m_{p}^{2}, \tilde{m}^{2},|t|}{Q\left|\tilde{\mathbf{p}}_{\perp}\right| H}\right)
$$

Thus small $x$ helps to keep the corrections small. On the other hand, very small $\left|\tilde{\mathbf{p}}_{\perp}\right|_{H}$, which corresponds to very small $|t|$, can lead to sizable corrections, as already noted above. We certainly recommend that experimentalists use the exact definitions of $\sin \varphi, \cos \varphi(2.16)$ whenever possible.

Coming back to the general case, we note that the angle $\varphi$ can only be defined unambiguously if the conditions

$$
\begin{aligned}
& -l^{2} \neq 0, \\
& -n^{2} \neq 0
\end{aligned}
$$

are satisfied $(\mathrm{cf} .(2.10))$. We see from $(2.15)$ that $(2.23)$ requires

$$
\left.\mathbf{q}_{\perp}\right|_{H}=-\left.\mathbf{k}_{\perp}^{\prime}\right|_{H} \neq 0,
$$

i.e. the scattered electron must have nonzero transverse momentum. Condition (2.24) requires for $s \rightarrow \infty$ that

$$
\left.\tilde{\mathbf{p}}_{\perp}\right|_{H} \neq 0,
$$

i.e. that the proton remnant has nonzero transverse momentum. The exact meaning of $n^{2} \neq 0$ for the case of arbitrary finite $s$ is discussed in appendix A.

\section{Cross sections and interference terms for defi- nite $\gamma^{*}$ helicities in the proton rest system}

Let us consider now in more detail the electroproduction reaction

$$
e^{\mp}(k)+p(p) \rightarrow e^{\mp}\left(k^{\prime}\right)+p(\tilde{p})+a_{1}\left(p_{1}\right)+\ldots+a_{n}\left(p_{n}\right) .
$$

Here we assume that the original proton is unpolarized, whereas we allow arbitrary polarization for the incoming electron or positron. The covariant density matrix of the initial $e^{-}$or $e^{+}$is then given by

$$
\left(\not k \pm m_{e}\right) \frac{1+\gamma_{5} \not}{2}
$$


where $s^{\mu}$ is the $e^{\mp}$ polarization vector which satisfies:

$$
\begin{aligned}
s^{\mu} k_{\mu} & =0 \\
-1 \leq s^{\mu} s_{\mu} & \leq 0 .
\end{aligned}
$$

For $m_{e} \rightarrow 0$ we can parametrize $s$ as follows:

$$
\begin{aligned}
& s \rightarrow r_{L} \frac{k}{m_{e}}+\left(\begin{array}{c}
0 \\
\mathbf{r}_{\perp}
\end{array}\right), \\
& \mathbf{r}_{\perp} \mathbf{k}=0, \\
& r_{L}^{2}+\mathbf{r}_{\perp}^{2} \leq 1 .
\end{aligned}
$$

Here the longitudinal and transverse polarizations are described by $r_{L}$ and $\mathbf{r}_{\perp}$, respectively, with $r_{L}=+1(-1)$ corresponding to a right(left)-handed electron or positron. We assume in this section that the proton remnant is again a single proton. The more general case of the remnant being a group of particles is treated in appendix $\mathrm{B}$. The hadrons $a_{1}, \ldots, a_{n}(n=1,2, .$.$) form the quasiparticle X$, where

$$
\sum_{i=1}^{n} p_{i}=p_{X}
$$

As is well known, the cross section for reaction (3.1) can be written as a product of a lepton and a hadron tensor

$$
d \sigma\left(e^{\mp} p \rightarrow e^{\mp} p X\right)=\frac{4 m_{p}}{s-m_{p}^{2}}\left(\frac{\alpha}{Q^{2}}\right)^{2} l^{\nu \mu} W_{\mu \nu}^{(X)} \frac{d^{3} k^{\prime}}{k^{\prime 0}} \frac{d^{3} \tilde{p}}{\tilde{p}^{0}}
$$

where (with the convention $\varepsilon_{0123}=1$ ):

$$
\begin{gathered}
l^{\nu \mu}=k^{\prime \nu} k^{\mu}+k^{\nu} k^{\prime \mu}-g^{\nu \mu}\left(k k^{\prime}-m_{e}^{2}\right)+i m_{e} \varepsilon^{\nu \mu \alpha \beta} q_{\alpha} s_{\beta} \\
\stackrel{m_{e} \rightarrow 0}{\longrightarrow} k^{\prime \nu} k^{\mu}+k^{\nu} k^{\prime \mu}-g^{\nu \mu}\left(k k^{\prime}\right)+i r_{L} \varepsilon^{\nu \mu \alpha \beta} q_{\alpha} k_{\beta}, \\
W_{\mu \nu}^{(X)}(p, q, \tilde{p})=\frac{1}{4 m_{p}} \sum_{n} \int_{p_{1}} \ldots \int_{p_{n}} \prod_{i=1}^{n} \frac{d^{3} p_{i}}{(2 \pi)^{3} 2 p_{i}^{0}} \\
\chi_{n}\left(p_{1}, \ldots, p_{n}, p, q, \tilde{p}\right) \delta\left(p+q-\tilde{p}-\sum_{i=1}^{n} p_{i}\right) \\
\sum_{\text {spins }}{ }^{\prime}<p(p)\left|J_{\mu}(0)\right| a_{i}\left(p_{i}\right), p(\tilde{p}) \text { out }><a_{i}\left(p_{i}\right), p(\tilde{p}) \text { out }\left|J_{\nu}(0)\right| p(p)>.
\end{gathered}
$$

Here and in the following $\sum_{\text {spins }}^{\prime}$ indicates the average and sum over the spin states of the initial and final state particles, respectively. The hadronic electromagnetic 
current is denoted by $e J_{\mu}$ and the functions $\chi_{n}$ are inserted as characteristic functions being 0 outside and 1 inside the region in phase space defining the set of final states $X$. The conventions for the normalization of states, for $\gamma$-matrices etc., are as in [18], in particular we set for a spinless particle $a(p)$ :

$$
<a\left(p^{\prime}\right) \mid a(p)>=(2 \pi)^{3} 2 p^{0} \delta^{3}\left(\mathbf{p}^{\prime}-\mathbf{p}\right) .
$$

The next step is to consider (3.1) as a reaction where a virtual photon $\gamma^{*}$ with density matrix $l^{\nu \mu}$ is produced which interacts then with the proton. We analyse now the helicity content of the density matrix $l^{\nu \mu}$ in the proton rest system. For this we define a vierbein of polarization vectors $\varepsilon_{\alpha}(\alpha=0,1,2,3)$ as follows:

$$
\begin{aligned}
\varepsilon_{0} & =\frac{(p \cdot q) q+Q^{2} p}{\sqrt{Q^{2}\left[(p \cdot q)^{2}+m_{p}^{2} Q^{2}\right]}}, \\
\varepsilon_{1} & =\frac{\eta}{\sqrt{-\eta^{2}}}, \\
\varepsilon_{2} & =\frac{n}{\sqrt{-n^{2}}}, \\
\varepsilon_{3} & =\frac{q}{\sqrt{-q^{2}}},
\end{aligned}
$$

where $n$ is defined in (2.8) and

$$
\eta=p_{X}-\frac{\left(p \cdot p_{X}\right)}{m_{p}^{2}} p+\frac{m_{p}^{2}\left(q \cdot p_{X}\right)-(p \cdot q)\left(p \cdot p_{X}\right)}{(p \cdot q)^{2}+m_{p}^{2} Q^{2}}\left[q-\frac{(p \cdot q)}{m_{p}^{2}} p\right] .
$$

In the rest system of the original proton with the coordinate axes as defined in Fig. 2 we have

$$
\begin{aligned}
&\left.\varepsilon_{0}\right|_{R}=\frac{1}{Q}\left(\begin{array}{c}
\sqrt{\nu^{2}+Q^{2}} \\
0 \\
0 \\
\nu
\end{array}\right), \\
&\left.\varepsilon_{1}\right|_{R}=\left(\begin{array}{l}
0 \\
1 \\
0 \\
0
\end{array}\right), \\
&\left.\varepsilon_{2}\right|_{R}=\left(\begin{array}{c}
0 \\
0 \\
1 \\
0
\end{array}\right), \\
&\left.\varepsilon_{3}\right|_{R}=\frac{1}{Q}\left(\begin{array}{c}
\nu \\
0 \\
0 \\
\sqrt{\nu^{2}+Q^{2}}
\end{array}\right) .
\end{aligned}
$$


From this we find easily

$$
\begin{aligned}
& \left(\varepsilon_{\alpha} \cdot \varepsilon_{\beta}\right)=g_{\alpha \beta}, \\
& \operatorname{det}\left(\varepsilon_{\alpha}, \varepsilon_{\beta}, \varepsilon_{\gamma}, \varepsilon_{\delta}\right)=\varepsilon_{\alpha \beta \gamma \delta} .
\end{aligned}
$$

The virtual photon $\gamma^{*}$ can have polarization vectors $\varepsilon_{0}$ and $\varepsilon_{1,2}$ corresponding to longitudinal and transverse polarizations, respectively, in the system $R$. The polarization vectors for right and left circular polarization are defined as

$$
\varepsilon_{ \pm}=\mp \frac{1}{\sqrt{2}}\left(\varepsilon_{1} \pm i \varepsilon_{2}\right)
$$

It is now straightforward to express the density matrix $l^{\nu \mu}$ in terms of the vectors $\varepsilon_{\alpha}$. We get

$$
\begin{aligned}
l^{\nu \mu}= & \frac{Q^{2}}{1-\varepsilon}\left\{\frac{1}{2}\left(\varepsilon_{+}^{\nu} \varepsilon_{+}^{\mu *}+\varepsilon_{-}^{\nu} \varepsilon_{-}^{\mu *}\right)+\varepsilon \varepsilon_{0}^{\nu} \varepsilon_{0}^{\mu *}\right. \\
& -\varepsilon \cos 2 \varphi \frac{1}{2}\left(\varepsilon_{-}^{\nu} \varepsilon_{+}^{\mu *}+\varepsilon_{+}^{\nu} \varepsilon_{-}^{\mu *}\right)+\varepsilon \sin 2 \varphi \frac{1}{2 i}\left(\varepsilon_{-}^{\nu} \varepsilon_{+}^{\mu *}-\varepsilon_{+}^{\nu} \varepsilon_{-}^{\mu *}\right) \\
& -\sqrt{\varepsilon(1+\varepsilon)} \cos \varphi \frac{1}{2}\left[\varepsilon_{0}^{\nu}\left(\varepsilon_{+}-\varepsilon_{-}\right)^{\mu *}+\left(\varepsilon_{+}-\varepsilon_{-}\right)^{\nu} \varepsilon_{0}^{\mu *}\right] \\
& +\sqrt{\varepsilon(1+\varepsilon)} \sin \varphi \frac{1}{2 i}\left[\varepsilon_{0}^{\nu}\left(\varepsilon_{+}+\varepsilon_{-}\right)^{\mu *}-\left(\varepsilon_{+}+\varepsilon_{-}\right)^{\nu} \varepsilon_{0}^{\mu *}\right] \\
& +r_{L} \sqrt{1-\varepsilon^{2}} \frac{1}{2}\left[\varepsilon_{+}^{\nu} \varepsilon_{+}^{\mu *}-\varepsilon_{-}^{\nu} \varepsilon_{-}^{\mu *}\right] \\
& -r_{L} \sqrt{\varepsilon(1-\varepsilon)} \cos \varphi \frac{1}{2}\left[\varepsilon_{0}^{\nu}\left(\varepsilon_{+}+\varepsilon_{-}\right)^{\mu *}+\left(\varepsilon_{+}+\varepsilon_{-}\right)^{\nu} \varepsilon_{0}^{\mu *}\right] \\
& \left.+r_{L} \sqrt{\varepsilon(1-\varepsilon)} \sin \varphi \frac{1}{2 i}\left[\varepsilon_{0}^{\nu}\left(\varepsilon_{+}-\varepsilon_{-}\right)^{\mu *}-\left(\varepsilon_{+}-\varepsilon_{-}\right)^{\nu} \varepsilon_{0}^{\mu *}\right]\right\}
\end{aligned}
$$

Here the quantity $\varepsilon$ is the usual ratio of longitudinal to transverse polarization strength of the $\gamma^{*}$ (cf. table 1$)$.

We define now the cross sections and interference terms for the absorption of virtual photons $\gamma^{*}$ of definite helicity in the system $R$ :

$$
\sigma_{m n}^{(X)}:=\frac{4 \pi^{2} \alpha}{K} \varepsilon_{m}^{* \mu} W_{\mu \nu}^{(X)} \varepsilon_{n}^{\nu} \quad(m, n=0, \pm 1)
$$

where we use Hand's convention [25] for the flux factor with $K$ as defined in table 1. It is clear that the matrix of these cross sections $\left(\sigma_{m n}^{(X)}\right)$ is hermitian and positive semi-definite. This means that we must have:

$$
\sigma_{m n}^{(X)}=\sigma_{n m}^{(X) *}
$$


and for an arbitrary complex vector $c=\left(c_{m}\right)$ :

$$
\sum_{m, n} c_{m}^{*} \sigma_{m n}^{(X)} c_{n} \geq 0 .
$$

These properties of the cross section matrix $\left(\sigma_{m n}^{(X)}\right)$ are discussed further in Appendix C. There we also show how the positivity condition (3.19) may be used to get information on the longitudinal cross section $\sigma_{00}^{(X)}$ - a quantity which is notoriously difficult to measure experimentally — from the measurements of the interference terms $\sigma_{m n}^{(X)}(m \neq n)$.

Inserting (3.16) and (3.17) in (3.7) we find

$$
\begin{aligned}
d \sigma\left(e^{\mp} p \rightarrow e^{\mp} p X\right)= & \Gamma\left\{\frac{1}{2}\left(\sigma_{++}^{(X)}+\sigma_{--}^{(X)}\right)+\varepsilon \sigma_{00}^{(X)}\right. \\
& -\varepsilon \cos (2 \varphi) \operatorname{Re} \sigma_{+-}^{(X)} \\
& +\varepsilon \sin (2 \varphi) \operatorname{Im} \sigma_{+-}^{(X)} \\
& -\sqrt{\varepsilon(1+\varepsilon)} \cos \varphi \operatorname{Re}\left(\sigma_{+0}^{(X)}-\sigma_{-0}^{(X)}\right) \\
& +\sqrt{\varepsilon(1+\varepsilon)} \sin \varphi \operatorname{Im}\left(\sigma_{+0}^{(X)}+\sigma_{-0}^{(X)}\right) \\
& +r_{L} \sqrt{1-\varepsilon^{2}} \frac{1}{2}\left(\sigma_{++}^{(X)}-\sigma_{--}^{(X)}\right) \\
& -r_{L} \sqrt{\varepsilon(1-\varepsilon)} \cos \varphi \operatorname{Re}\left(\sigma_{+0}^{(X)}+\sigma_{-0}^{(X)}\right) \\
& \left.+r_{L} \sqrt{\varepsilon(1-\varepsilon)} \sin \varphi \operatorname{Im}\left(\sigma_{+0}^{(X)}-\sigma_{-0}^{(X)}\right)\right\} \\
& \cdot \frac{m_{p}}{\left(p k^{\prime}\right)} \frac{d^{3} k^{\prime}}{k^{\prime}} \frac{d^{3} \tilde{p}}{\tilde{p}^{0}},
\end{aligned}
$$

where $\Gamma$ is the conventional flux factor of virtual photons (cf. [21):

$$
\Gamma=\frac{\alpha}{2 \pi^{2}} \frac{\left(p \cdot k^{\prime}\right)}{(p \cdot k)} \frac{W^{2}-m_{p}^{2}}{2 m_{p} Q^{2}} \frac{1}{1-\varepsilon} .
$$

So far the analysis is completely general, valid for any set of final states $X$.

Now we will consider the case that the final state $X$ is defined according to criterion I or II of sect. 2. Choosing criterion I (2.2) we have to set for the functions $\chi_{n}$ in (3.9)

$$
\chi_{n}^{(I)}\left(p_{1}, \ldots, p_{n}, p, q, \tilde{p}\right)=\Theta\left(\xi_{0}-\frac{(p-\tilde{p}) \cdot q}{p \cdot q}\right) .
$$

Here $\Theta(\cdot)$ is the usual step function. For criterion II the relevant condition is given in (2.3). This is implemented by setting for the functions $\chi_{n}$ in (3.9):

$$
\chi_{n}^{(I I)}\left(p_{1}, \ldots, p_{n}, p, q, \tilde{p}\right)=\prod_{j=1}^{n} \Theta\left[M_{c u t}^{2}-p_{j} \cdot(p+q-\tilde{p})\right] .
$$


In both cases, (3.22) and (3.23) we can treat $X$ as a quasi particle with $W_{\mu \nu}^{(X)}$ depending only on the 4-momenta $p, q, \tilde{p}$ :

$$
W_{\mu \nu}^{(X)}=W_{\mu \nu}^{(X)}(p, q, \tilde{p}) .
$$

We note that the interference terms $\sigma_{m n}^{(X)}(m \neq n)$ must vanish for $t=-|t|_{\text {min }}$ and $t=-|t|_{\max }$ (cf. Appendix A). For these $t$ values the reaction $\gamma^{*} p \rightarrow p X$ is collinear in the original proton rest system $R$. With any of the criteria I or II the final state $p X$ is then rotationally invariant around the collision axis, which implies by an elementary angular momentum conservation argument:

$$
\sigma_{m n}^{(X)}=0 \quad \text { for } \quad t=-|t|_{\text {min }}, \quad \text { and } \quad t=-|t|_{\max } \quad \text { if } \quad m \neq n .
$$

Due to (3.25) the $\varphi$-dependent terms in (3.20) vanish for the collinear situation and thus the fact that the angle $\varphi$ becomes undefined there (cf. (2.24)) causes no problems.

The functions $\chi_{n}^{(I, I I)},(3.22)$ and $(\overline{3.23})$, are parity-invariant. Then parity invariance of the strong interactions gives the condition:

$$
W_{\mu \nu}^{(X)}(p, q, \tilde{p})=W^{(X) \mu \nu}\left(p^{\prime}, q^{\prime}, \tilde{p}^{\prime}\right),
$$

where the parity transformed 4-momenta are:

$$
p^{\prime \mu}=p_{\mu}, q^{\prime \mu}=q_{\mu}, \tilde{p}^{\prime \mu}=\tilde{p}_{\mu} .
$$

Furthermore we have from (3.11)-(3.15)

$$
\varepsilon_{m}^{\mu}\left(p^{\prime}, q^{\prime}, \tilde{p}^{\prime}\right)=(-1)^{m} \varepsilon_{-m \mu}(p, q, \tilde{p}) \quad(m=0, \pm 1) .
$$

From (3.17) and (3.24) we find that the cross sections $\sigma_{m n}^{(X)}$ are Lorentz-invariant functions of the momenta $p, q, \tilde{p}$ and thus only functions of the invariants we can form from $p, q, \tilde{p}$. There are 4 such invariants, all parity even, a convenient set being (cf. table 1)

$$
x, Q^{2}, \xi, t
$$

We get then

$$
\sigma_{m n}^{(X)}=\sigma_{m n}^{(X)}\left(x, Q^{2}, \xi, t\right) .
$$

From parity invariance (3.26) and (3.28) we get now:

$$
\sigma_{m n}^{(X)}\left(x, Q^{2}, \xi, t\right)=(-1)^{m+n} \sigma_{-m,-n}^{(X)}\left(x, Q^{2}, \xi, t\right) .
$$

From (3.18) and (3.31) we find the following relations:

$$
\begin{aligned}
\sigma_{++}^{(X)} & =\sigma_{--}^{(X)}, \\
\sigma_{+-}^{(X)} & =\sigma_{-+}^{(X)}=\sigma_{+-}^{(X) *} \\
\sigma_{+0}^{(X)} & =-\sigma_{-0}^{(X)} .
\end{aligned}
$$


The last step is to insert (3.32) into the general formula (3.20) and to express the phase space element in terms of the variables

$$
x, Q^{2}, \xi, t, \varphi, \tilde{\psi}
$$

where $\tilde{\psi}$ is the azimuthal angle of $\tilde{\mathbf{p}}$ in the HERA system (Fig. 4 ) with respect to a fixed laboratory frame. We get:

$$
\begin{aligned}
& \frac{d^{3} k^{\prime}}{k^{\prime}} \frac{d^{3} \tilde{p}}{\tilde{p}^{0}}=\frac{y}{4 x}\left(1+\frac{4 x^{2} m_{p}^{2}}{Q^{2}}\right)^{-1 / 2} d x d Q^{2} d \xi d t d \varphi d \tilde{\psi} \\
& \frac{d^{6} \sigma(e p \rightarrow e p X)}{d x d Q^{2} d \xi d t d \varphi d \tilde{\psi}}=\frac{1}{2 \pi} \tilde{\Gamma}\left\{\sigma_{++}^{(X)}+\varepsilon \sigma_{00}^{(X)}-\varepsilon \cos (2 \varphi) \sigma_{+-}^{(X)}\right. \\
& \left.-2 \sqrt{\varepsilon(1+\varepsilon)} \cos \varphi \operatorname{Re} \sigma_{+0}^{(X)}+2 r_{L} \sqrt{\varepsilon(1-\varepsilon)} \sin \varphi \operatorname{Im} \sigma_{+0}^{(X)}\right\} .
\end{aligned}
$$

The flux factor $\tilde{\Gamma}$ is given by

$$
\begin{aligned}
& \tilde{\Gamma}=\frac{\pi m_{p} y^{3 / 2}}{2 x\left(p k^{\prime}\right)}\left(y+\frac{4 x m_{p}^{2}}{s-m_{p}^{2}}\right)^{-1 / 2} \cdot \Gamma \\
& =\frac{\alpha}{2 \pi\left(s-m_{p}^{2}\right)} \frac{m_{p} K y^{3 / 2}}{x Q^{2}(1-\varepsilon)}\left(y+\frac{4 x m_{p}^{2}}{s-m_{p}^{2}}\right)^{-1 / 2} .
\end{aligned}
$$

The right-hand side of (3.34) is, of course, independent of the overall azimuthal angle $\tilde{\psi}$ in the HERA system. To measure the cross section (3.34) and in particular the $\varphi$ dependence we think of an experimental setup where the scattered proton $p(\tilde{p})$ is detected with the help of a small angle forward detector having some azimuthal coverage $\Delta \tilde{\psi}$. The relative angle $\varphi^{\prime}$ (cf. Fig. 4) which is simply related to $\varphi$ (cf. (2.16)(2.20) ) should then easily be measurable since the ZEUS and H1 detectors have full azimuthal coverage for the scattered lepton. Integrating (3.34) over $\tilde{\psi}$ in the interval $\Delta \tilde{\psi}$ we get

$$
\begin{aligned}
\frac{d^{5} \sigma(e p \rightarrow e p X)}{d x d Q^{2} d \xi d t d \varphi}= & \frac{\Delta \tilde{\psi}}{2 \pi} \tilde{\Gamma}\left\{\sigma_{++}^{(X)}+\varepsilon \sigma_{00}^{(X)}-\varepsilon \cos (2 \varphi) \sigma_{+-}^{(X)}\right. \\
& -2 \sqrt{\varepsilon(1+\varepsilon)} \cos \varphi \operatorname{Re} \sigma_{+0}^{(X)} \\
& \left.+2 r_{L} \sqrt{\varepsilon(1-\varepsilon)} \sin \varphi \operatorname{Im} \sigma_{+0}^{(X)}\right\}
\end{aligned}
$$

The case where the scattered proton is not detected and the scattered lepton is accepted over the full azimuth corresponds to integrating over $\varphi$ and $\tilde{\psi}$ in (3.34). We get then

$$
\frac{d^{4} \sigma(e p \rightarrow e p X)}{d x d Q^{2} d \xi d t}=2 \pi \tilde{\Gamma}\left(\sigma_{++}^{(X)}+\varepsilon \sigma_{00}^{(X)}\right)
$$


which allows us to make easy contact with the conventional notation for this 4dimensional cross section [6], [7]. Let us define

$$
\begin{aligned}
& F_{2}^{D(4)}\left(x, Q^{2}, \xi, t\right):=\frac{Q^{2}(1-x)}{4 \pi \alpha}\left(1+\frac{4 x^{2} m_{p}^{2}}{Q^{2}}\right)^{-3 / 2}\left(\sigma_{++}^{(X)}+\sigma_{00}^{(X)}\right), \\
& R^{D(4)}\left(x, Q^{2}, \xi, t\right):=\frac{\sigma_{00}^{(X)}}{\sigma_{++}^{(X)}} .
\end{aligned}
$$

We get then from (3.37) the usual expression:

$$
\begin{aligned}
\frac{d^{4} \sigma(e p \rightarrow e p X)}{d x d Q^{2} d \xi d t}= & \frac{4 \pi \alpha^{2}}{x Q^{4}}\left\{1-y-\frac{m_{p}^{2} x y}{s-m_{p}^{2}}\right. \\
& \left.+\frac{1}{2}\left[y^{2}+\frac{4 x y m_{p}^{2}}{s-m_{p}^{2}}\right]\left[1+R^{D(4)}\right]^{-1}\right\} F_{2}^{D(4)}
\end{aligned}
$$

The formula (3.34) is the main result of this section. Note that in (3.34) the dependence of the cross section on the azimuthal angle $\varphi$ is completely explicit. The coefficients $\sigma_{++}^{(X)}, \sigma_{00}^{(X)}$ etc. depend only on $x, Q^{2}, \xi, t(\operatorname{cf}$. (3.30)). The neat separation of $\varphi$ from these latter kinematic variables which describe the reaction $\gamma^{*} p \rightarrow p X$ is due to the fact that (a) we chose to analyse the reaction (3.1) in the proton rest system $R$ with respect to $\mathbf{q}$ as 3 -axis, and (b) we imposed the criterion I (2.2) or criterion II (2.3) for defining $X$ which allowed us to treat $X$ as a quasi particle. In this respect our analysis differs in a fundamental way from the analysis presented in [26] where instead of the $\mathbf{q}$ axis in $R$ the axis defined by the electron and proton beams in the HERA system is used to define $X$ and the kinematic variables, in particular an azimuthal angle. Not surprisingly, in the latter case the kinematic dependence on their azimuthal angle is coupled with the dynamic dependence on the momentum transfer squared $t$ of reaction (1.3). We think it is fair to say that the analysis of [26] may be more easily implemented experimentally whereas the analysis proposed in our paper requires more experimental work but - as we hope to show below - has the promise of rendering more directly interesting information on the dynamics of the diffractive reaction (1.3), namely on the helicity structure of the "Pomeron".

\section{The helicity structure of the reaction $\gamma^{*} p \rightarrow p X$ in the rest system of $X$}

In this section we propose to look at reaction (3.1) in the rest system of $X$, denoted by $R X$ in the following. We will define cross sections and interference terms for the various helicities of $\gamma^{*}$ in the system $R X$. 
We start by defining a coordinate system in $R X$ with the $\gamma^{*} 3$-momentum $\left.\mathbf{q}\right|_{R X}$ as 3-axis and the plane spanned by $\left.\mathbf{q}\right|_{R X}$ and $\left.\mathbf{p}\right|_{R X}$ as 1-3 plane (Fig. 5). We define a vierbein of polarization vectors $\tilde{\varepsilon}_{\alpha}(\alpha=0,1,2,3)$ related to the system $R X$ as in (3.11) but with the replacements:

$$
p \rightarrow p_{X}, m_{p} \rightarrow m_{X}, p_{X} \rightarrow p
$$

This leads to:

$$
\begin{aligned}
& \tilde{\varepsilon}_{0}=\frac{\left(p_{X} \cdot q\right) q+Q^{2} p_{X}}{\sqrt{Q^{2}\left[\left(p_{X} \cdot q\right)^{2}+Q^{2} m_{X}^{2}\right]}} \\
& \tilde{\varepsilon}_{1}=\frac{\tilde{\eta}}{\sqrt{-\tilde{\eta}^{2}}} \\
& \tilde{\varepsilon}_{2}=\frac{\tilde{n}}{\sqrt{-\tilde{n}^{2}}}, \\
& \tilde{\varepsilon}_{3}=\frac{q}{\sqrt{-q^{2}}}
\end{aligned}
$$

where

$$
\begin{aligned}
\tilde{\eta} & =p-\frac{\left(p_{X} \cdot p\right)}{m_{X}^{2}} p_{X}+\frac{m_{X}^{2}(q \cdot p)-\left(p_{X} \cdot q\right)\left(p_{X} \cdot p\right)}{\left(p_{X} \cdot q\right)^{2}+Q^{2} m_{X}^{2}}\left[q-\frac{\left(p_{X} \cdot q\right)}{m_{X}^{2}} p_{X}\right] \\
\tilde{n}^{\mu} & =\frac{1}{m_{X}} \varepsilon^{\mu \nu \rho \sigma} p_{X \nu} q_{\rho} p_{\sigma}=-\frac{m_{p}}{m_{X}} n^{\mu} .
\end{aligned}
$$

We have as in (3.14)

$$
\begin{aligned}
& \left(\tilde{\varepsilon}_{\alpha} \cdot \tilde{\varepsilon}_{\beta}\right)=g_{\alpha \beta}, \\
& \operatorname{det}\left(\tilde{\varepsilon}_{\alpha}, \tilde{\varepsilon}_{\beta}, \tilde{\varepsilon}_{\gamma}, \tilde{\varepsilon}_{\delta}\right)=\varepsilon_{\alpha \beta \gamma \delta} .
\end{aligned}
$$

The vectors $\varepsilon_{\alpha}$ and $\tilde{\varepsilon}_{\alpha}$ are related by a proper, orthochronous Lorentz transformation:

$$
\tilde{\varepsilon}_{\alpha}=\varepsilon_{\beta} \Lambda_{\alpha}^{\beta} .
$$

A simple calculation gives for $\Lambda_{\alpha}^{\beta}$ :

$$
\left(\Lambda_{\alpha}^{\beta}{ }_{\alpha}\right)=\left(\begin{array}{cccc}
\gamma_{X} & -v_{X} \gamma_{X} & 0 & 0 \\
v_{X} \gamma_{X} & -\gamma_{X} & 0 & 0 \\
0 & 0 & -1 & 0 \\
0 & 0 & 0 & 1
\end{array}\right)
$$

where 


$$
\begin{aligned}
\gamma_{X} & =\left[\left(p_{X} \cdot p\right)(p \cdot q)+Q^{2}\left(p_{X} \cdot p\right)\right]\left[(p \cdot q)^{2}+m_{p}^{2} Q^{2}\right]^{-1 / 2}\left[\left(p_{X} \cdot q\right)^{2}+m_{X}^{2} Q^{2}\right]^{-1 / 2} \\
& =\left(1+\frac{4 x^{2} m_{p}^{2}}{Q^{2}}\right)^{-1 / 2}\left(1+\frac{4 \beta^{2} t}{Q^{2}}\right)^{-1 / 2}\left(1+\frac{2 \beta^{2} \xi t}{Q^{2}}\right) \\
v_{X} & =\left(1-\gamma_{X}^{-2}\right)^{1 / 2} \\
& =\frac{2 \beta}{Q}\left[-t(1-\xi)-m_{p}^{2} \xi^{2}-\frac{t\left(4 m_{p}^{2}-t\right) x^{2}}{Q^{2}}\right]^{1 / 2}\left(1+\frac{2 \beta^{2} \xi t}{Q^{2}}\right)^{-1}
\end{aligned}
$$

The boost velocity $v_{X}$ is always small and the Lorentz factor $\gamma_{X}$ close to one for the kinematic range of interest at HERA:

$$
v_{X} \approx \frac{2 \beta \sqrt{-t}}{Q} \ll 1 .
$$

We define now the vectors for right and left circular polarization of $\gamma^{*}$ in the system $R X$ :

$$
\tilde{\varepsilon}_{ \pm}=\mp \frac{1}{\sqrt{2}}\left(\tilde{\varepsilon}_{1} \pm i \tilde{\varepsilon}_{2}\right)
$$

and the cross sections and interference terms

$$
\tilde{\sigma}_{m n}^{(X)}=\frac{4 \pi^{2} \alpha}{K} \tilde{\varepsilon}_{m}^{\mu *} W_{\mu \nu}^{(X)} \tilde{\varepsilon}_{n}^{\nu} \quad(m, n=0, \pm) .
$$

The linear relation between the $\tilde{\sigma}_{m n}^{(X)}$ and the $\sigma_{m n}^{(X)}$ is given by a $5 \times 5$ matrix $\mathcal{M}$ for the case which we consider here, i.e. where (3.32) holds for $\sigma_{m n}^{(X)}$ and, as is easily seen, also for $\tilde{\sigma}_{m n}^{(X)}$ :

$$
\left(\begin{array}{l}
\tilde{\sigma}_{++}^{(X)} \\
\tilde{\sigma}_{00}^{(X)} \\
\tilde{\sigma}_{+-}^{(X)} \\
\operatorname{Re} \tilde{\sigma}_{+0}^{(X)} \\
\operatorname{Im} \tilde{\sigma}_{+0}^{(X)}
\end{array}\right)=\mathcal{M}\left(\begin{array}{l}
\sigma_{++}^{(X)} \\
\sigma_{00}^{(X)} \\
\sigma_{+-}^{(X)} \\
\operatorname{Re} \sigma_{+0}^{(X)} \\
\operatorname{Im} \sigma_{+0}^{(X)}
\end{array}\right)
$$

The matrix $\mathcal{M}$ is given in table 3 .

\section{Expectations for $\tilde{\sigma}_{m n}^{(X)}$ in some models for the reaction $\gamma^{*} p \rightarrow p X$}

In this section we discuss the expectations one can have for the quantities $\tilde{\sigma}_{m n}^{(X)}(4.10$ in various models for the reaction $\gamma^{*} p \rightarrow p X$. 


\subsection{Pomeron exchange models with factorization}

Let us start by discussing the class of models where the reaction (1.3) is viewed as a two-step process: The original proton emits a Pomeron which collides with the $\gamma^{*}$ to give the final hadronic state $X$ :

$$
\begin{gathered}
p(p) \rightarrow p(\tilde{p})+\mathbb{P}(\Delta), \\
\gamma^{*}(q)+\mathbb{P}(\Delta) \rightarrow X\left(p_{X}\right) .
\end{gathered}
$$

By (5.1) and (5.2) we certainly do not want to imply that the Pomeron is a particle but only that the amplitude for (1.3) can be written in a factorizable form:

$$
A\left(\gamma^{*} p \rightarrow f p\right)=A\left(\gamma^{*} \mathbb{P} \rightarrow f\right) \otimes(\mathbb{P}-\text { propagator }) \otimes(p p \mathbb{P}-\text { vertex factor })
$$

where $f$ is some final state of $X(f \in X)$. The proton-proton Pomeron vertex factor is allowed to have arbitrary Lorentz structure, i.e. it can be the sum of a scalar, a vector, and tensors of arbitrary rank. Writing out (5.3) explicitly we have then:

$$
\begin{aligned}
<f, p(\tilde{p}) \text { out }\left|J_{\mu}(0)\right| p(p)>= & \mathcal{M}_{\mu}^{f} \bar{u}(\tilde{p}) \Gamma u(p)+\mathcal{M}_{\mu, \alpha}^{f} \bar{u}(\tilde{p}) \Gamma^{\alpha} u(p) \\
& +\mathcal{M}_{\mu, \alpha_{1}, \alpha_{2}}^{f} \bar{u}(\tilde{p}) \Gamma^{\alpha_{1}, \alpha_{2}} u(p)+\ldots
\end{aligned}
$$

Here $\Gamma, \Gamma^{\alpha}, \ldots$ stand for $\mathbb{P}$ propagator times $p p \mathbb{P}$ vertex factor and $\mathcal{M}_{\mu}^{f}, \mathcal{M}_{\mu, \alpha}^{f}, \ldots$ for the $\gamma^{*} \mathbb{P} \rightarrow f$ amplitudes. We want to consider all models where the following two hypotheses hold:

(1) The amplitudes $\mathcal{M}_{\mu}^{f}, \mathcal{M}_{\mu, \alpha}^{f}, \ldots$ depend only on the momenta $q, \Delta$ and the momenta and spins of the particles in the final state $f$.

(2) In contrast, the $\mathbb{P} p p$-vertex times $\mathbb{P}$-propagator factors $\Gamma, \Gamma^{\alpha}, \ldots$ depend only on the momenta $p, \tilde{p}$ of the incoming and scattered proton and on the variable $\xi$. The typical "effective $\mathbb{P}$-propagator" in Regge theory has a $\xi$ dependence (cf. next section) and thus we allow for it in (5.4).

With the above we have given a precise definition of what we call the Pomeron factorization property. In the following we will show how one can test some aspects of such a factorizing Pomeron.

An explicit example of a model with factorizing Pomeron is the DonnachieLandshoff model [9], which we will discuss at length in section 5.2. Typically in this type of model $\mathbb{P}$-exchange is built up from a whole series of spin $n=2,4,6, \ldots$ exchanges. The coherent sum of these exchanges can give an effective vector-type coupling of the Pomeron as was exemplified explicitly in a model due to Van Hove [27 a long time ago (cf. also chapt. 6.2 of [13]). We also note the following: The propagator in the usual field theoretic sense for one of the exchanged particles of definite spin (e.g. a glueball of spin $n$ ) depends, of course, only on the momentum $\Delta$. The corresponding propagator times vertex factor $\Gamma^{\alpha_{1}, \ldots, \alpha_{n}}$ in (5.4) has then no 
dependence on $q$ and thus no $\xi$ dependence. The latter is introduced only by the contraction with $\mathcal{M}_{\mu, \alpha_{1}, \ldots, \alpha_{n}}^{f}$. When the sum over $n$ is performed one gets Pomeron exchange as an effective vector exchange with a Pomeron "Propagator" depending on $\xi$.

In the rest system of $X, R X$ (Fig. 5), the $\gamma^{*}-\mathbb{P}$ collision is head on. With the criteria I or II of sect. 2 the set $X$ of final states is invariant with respect to rotations around the axis given by the $\mathbb{P}$-momentum $\boldsymbol{\Delta}_{R X}$. We imagine now that we go from the Lorentz indices $\alpha, \alpha_{1}, \alpha_{2} \ldots$ in (5.4) to a helicity basis for the Pomeron with respect to the axis $\boldsymbol{\Delta}_{R X}$. Then (5.4) can be rewritten in the form:

$$
<f, p(\tilde{p}) \text { out }\left|J_{\mu}(0)\right| p(p)>=\mathcal{M}_{\mu r}^{f} \bar{u}(\tilde{p}) \Gamma_{r} u(p)
$$

where $r=0, \pm 1, \pm 2, \ldots$ are the helicity indices of $\mathbb{P}$. (N.B.: The summation convention is always used.) Inserting (5.5) in (3.9) and (4.10) we get:

$$
\begin{gathered}
W_{\mu \nu}^{(X)}=S_{\mu r, \nu s} \rho_{s r}, \\
\tilde{\sigma}_{m n}^{(X)}=\frac{4 \pi^{2} \alpha}{K} S_{m r, n s} \rho_{s r},
\end{gathered}
$$

where

$$
\begin{aligned}
S_{\mu r, \nu s} & =\frac{1}{2 m_{p}} \sum_{f \in X} \delta\left(\Delta+q-p_{f}\right) \mathcal{M}_{\mu r}^{f *} \mathcal{M}_{\nu s}^{f}, \\
S_{m r, n s} & =\tilde{\varepsilon}_{m}^{\mu *} S_{\mu r, \nu s} \tilde{\varepsilon}_{n}^{\nu}, \\
\rho_{s r} & =\frac{1}{2} \sum_{\text {spins }}{ }^{\prime} \bar{u}(\tilde{p}) \Gamma_{s} u(p) \bar{u}(p) \bar{\Gamma}_{r} u(\tilde{p}) .
\end{aligned}
$$

Clearly, $\left(\rho_{s r}\right)$ can be considered as the Pomeron density matrix in the helicity basis in much the same way as the lepton tensor $l^{\nu \mu}$ was considered as density matrix of the virtual photon in sect. 3 .

We can state the following:

Theorem: If the Pomeron is described in the system $R X$ by a density matrix $\left(\rho_{s r}\right)$ which is diagonal in helicity, then the interference terms $\tilde{\sigma}_{m n}^{(X)}, m \neq n$, must vanish, i.e.

$$
\begin{aligned}
& \tilde{\sigma}_{+-}^{(X)}=0, \\
& \tilde{\sigma}_{+0}^{(X)}=0 .
\end{aligned}
$$

The proof of this theorem follows immediately by exploiting rotational invariance around the common axis of $\mathbf{q}$ and $\boldsymbol{\Delta}=-\mathbf{q}$ in $R X$ (Fig. 5). The quantity $S_{m r, n s}$ (5.8) can be considered as the generalized "absorptive part" of an amplitude for a "reaction"

$$
\gamma^{*} \mathbb{P} \rightarrow X \rightarrow \gamma^{*} \mathbb{P}
$$


where one does not sum over a complete set of intermediate states, but only over a set $X$ selected by our criteria I or II of sect. 2 . Now it is important that this set is invariant under rotations around $\boldsymbol{\Delta}_{R X}$. Then the angular momentum around $\boldsymbol{\Delta}_{R X}$ for $\gamma^{*} \mathbb{P}$ in the initial state of (5.11): $s-n$ must be equal to the one in the final state: $r-m$ (cf. appendix $\mathrm{D}$ for more details). Thus we get

$$
S_{m r, n s}=0 \text { for } m-r \neq n-s .
$$

If we have a diagonal $\mathbb{P}$-density matrix, i.e. if

$$
\rho_{s r}=0 \quad \text { for } \quad r \neq s
$$

we get from (5.7) and (5.12)

$$
\tilde{\sigma}_{m n}^{(X)}=0 \text { for } m \neq n,
$$

q.e.d.

What can we learn from the theorem (5.10)? If $\tilde{\sigma}_{+-}^{(X)}$ and/or $\tilde{\sigma}_{+0}^{(X)}$ are found to be different from zero in experiment, we learn in the framework of the $\mathbb{P}$-exchange models that the Pomeron is not in a diagonal helicity state. This would imply that the Pomeron cannot have a single helicity, e.g. helicity zero only. The Pomeron density matrix would have to contain non-diagonal terms in helicity. More generally, going beyond the ansatz of a factorizing Pomeron (5.3), we would learn that the information on the azimuthal orientation of the $\mathbf{p}-\tilde{\mathbf{p}}$ plane in Fig. 5 relative to the polarization directions 1,2 of the photon $\gamma^{*}$ must have reached the system $X$. This could have happened through the initial state, e.g. by a nondiagonal helicity density matrix of the exchanged object, as discussed above, and/or by final state interactions between the remnant $p(\tilde{p})$ and the system $X$. The latter possibility will be discussed in more detail below in sect. 5.3.

\subsection{The Donnachie-Landshoff Pomeron}

In this section we explore the consequences of the Donnachie-Landshoff Pomeron model [3, 9] for the cross sections and interference terms $\tilde{\sigma}_{m n}^{(X)}$ defined in (4.10). In this model $\mathbb{P}$-exchange is viewed as exchange of a " $C=+1$ photon". The $p p \mathbb{P}$ vertex factor in Fig. 5 is taken to be proportional to the one for an isoscalar photon and the "P्p-propagator" $P(\xi)$ has the form [3]:

$$
P(\xi)=(\xi)^{1-\alpha_{\mathbb{P}}(t)} \otimes \text { phase factor. }
$$

Here $\alpha_{\mathbb{P}}(t)$ is the Pomeron-trajectory, determined experimentally in hadron-hadron scattering as

$$
\begin{aligned}
& \alpha_{I P}(t)=1+\varepsilon+\alpha^{\prime} t, \\
& \varepsilon=0.08 \text { to } 0.09, \\
& \alpha^{\prime}=0.25 \mathrm{GeV}^{-2} .
\end{aligned}
$$


The ansatz for $\gamma^{*} p \rightarrow p f$ in this model is then (cf. (5.4))

$$
\begin{array}{r}
<f, p(\tilde{p}) \text { out }\left|J_{\mu}(0)\right| p(p)>=\mathcal{M}_{\mu \alpha}^{f} P(\xi) i 3 \beta_{q} \\
\cdot \bar{u}(\tilde{p})\left[F_{1}(t) \gamma^{\alpha}-\frac{i}{2 m_{p}} \sigma^{\alpha \beta} \Delta_{\beta} F_{2}(t)\right] u(p) .
\end{array}
$$

Here

$$
\beta_{q} \simeq 1.8 \mathrm{GeV}^{-1}
$$

is the quark-Pomeron coupling constant and $F_{1,2}$ are the isoscalar Dirac and Pauli electromagnetic nucleon form factors, the sum of the proton and neutron form factors:

$$
F_{j}(t)=F_{j}^{p}(t)+F_{j}^{n}(t) \quad(j=1,2) .
$$

A simple calculation gives now

$$
W_{\mu \nu}^{(X)}=S_{\mu \alpha, \nu \beta} \rho^{\beta \alpha},
$$

where

$$
\begin{aligned}
& S_{\mu \alpha, \nu \beta}=\frac{1}{2 m_{p}} \sum_{f \in X} \delta\left(\Delta+q-p_{f}\right) \mathcal{M}_{\mu \alpha}^{f *} \mathcal{M}_{\nu \beta}^{f}, \\
& \rho^{\beta \alpha}=9 \beta_{q}^{2}|P(\xi)|^{2} \tilde{\rho}^{\beta \alpha}, \\
& \tilde{\rho}^{\beta \alpha}=\frac{1}{2} \sum_{\text {spins }}{ }^{\prime} \bar{u}(\tilde{p})\left[F_{1} \gamma^{\beta}-\frac{i}{2 m_{p}} \sigma^{\beta \beta^{\prime}} \Delta_{\beta^{\prime}} F_{2}\right] u(p) \\
& \bar{u}(p)\left[F_{1} \gamma^{\alpha}+\frac{i}{2 m_{p}} \sigma^{\alpha \alpha^{\prime}} \Delta_{\alpha^{\prime}} F_{2}\right] u(\tilde{p}) \\
& =2\left(p^{\beta}-\frac{(p \cdot \Delta) \Delta^{\beta}}{t}\right)\left(p^{\alpha}-\frac{(p \cdot \Delta) \Delta^{\alpha}}{t}\right) \tilde{G}^{2}(t) \\
& +\frac{t}{2}\left(g^{\beta \alpha}-\frac{\Delta^{\beta} \Delta^{\alpha}}{t}\right) G_{M}^{2}(t), \\
& G_{E}(t)=F_{1}(t)+\frac{t}{4 m_{p}^{2}} F_{2}(t), \\
& G_{M}(t)=F_{1}(t)+F_{2}(t), \\
& \tilde{G}(t)=\left(G_{E}^{2}(t)-\frac{t}{4 m_{p}^{2}} G_{M}^{2}(t)\right)^{1 / 2}\left(1-\frac{t}{4 m_{p}^{2}}\right)^{-1 / 2} .
\end{aligned}
$$


The form factors $G_{E}$ and $G_{M}$ are the usual electric and magnetic isoscalar Sachs form factors. For $t=0$ we have

$$
\begin{aligned}
G_{E}(0) & =\tilde{G}(0)=1 \\
G_{M}(0) & =\left(\mu_{p}+\mu_{n}\right) /\left(\frac{e}{2 m_{p}}\right) \simeq 0.88
\end{aligned}
$$

where $\mu_{p}\left(\mu_{n}\right)$ is the magnetic moment of the proton (neutron). Data for the form factors at values $t<0$ are compiled in 28].

The problem is now to represent $\tilde{\rho}^{\beta \alpha}$ in the helicity basis. This is easily achieved by introducing a vierbein of $\mathbb{P}$-polarization vectors $\zeta_{\lambda}(\lambda=0,1,2,3)$ in analogy to (3.11)-(3.13), but with the replacements

$$
p \rightarrow p_{X}, q \rightarrow \Delta, p_{X} \rightarrow p
$$

which imply, of course, $p^{2}=m_{p}^{2} \rightarrow p_{X}^{2}=m_{X}^{2}$. This leads to

$$
\begin{aligned}
\zeta_{0} & =\frac{\left(p_{X} \cdot \Delta\right) \Delta-t p_{X}}{\sqrt{-t\left[\left(p_{X} \cdot \Delta\right)^{2}-m_{X}^{2} t\right]}}, \\
\zeta_{1} & =\frac{\zeta_{1}^{\prime}}{\sqrt{-\zeta_{1}^{\prime 2}}}, \\
\zeta_{2} & =\frac{\zeta_{2}^{\prime}}{\sqrt{-\zeta_{2}^{\prime 2}}}, \\
\zeta_{3} & =\frac{\Delta}{\sqrt{-t}},
\end{aligned}
$$

where

$$
\begin{aligned}
\zeta_{1}^{\prime}= & p-\frac{\left(p_{X} \cdot p\right)}{m_{X}^{2}} p_{X} \\
& +\frac{m_{X}^{2}(\Delta \cdot p)-\left(p_{X} \cdot \Delta\right)\left(p_{X} \cdot p\right)}{\left(p_{X} \cdot \Delta\right)^{2}-m_{X}^{2} t}\left[\Delta-\frac{\left(p_{X} \cdot \Delta\right)}{m_{X}^{2}} p_{X}\right], \\
\zeta_{2}^{\prime \mu}= & \frac{1}{m_{X}} \varepsilon^{\mu \nu \rho \sigma} p_{X \nu} \Delta_{\rho} p_{\sigma} .
\end{aligned}
$$

The vectors $\zeta_{\lambda}$ satisfy

$$
\begin{aligned}
\left(\zeta_{\kappa} \cdot \zeta_{\lambda}\right) & =g_{\kappa \lambda}, \\
\operatorname{det}\left(\zeta_{0}, \zeta_{1}, \zeta_{2}, \zeta_{3}\right) & =1 .
\end{aligned}
$$


In the rest system of $X$ we have with $\widehat{\Delta}=\Delta /|\Delta|$ :

$$
\begin{aligned}
\left.\zeta_{0}\right|_{R X} & =\frac{1}{\sqrt{-t}}\left(\begin{array}{c}
|\boldsymbol{\Delta}| \\
\Delta^{0} \widehat{\boldsymbol{\Delta}}
\end{array}\right)_{R X}, \\
\left.\zeta_{1}\right|_{R X} & =\left(\begin{array}{c}
0 \\
\frac{\mathbf{p}-\widehat{\boldsymbol{\Delta}}(\widehat{\boldsymbol{\Delta}} \cdot \mathbf{p})}{|\mathbf{p}-\widehat{\boldsymbol{\Delta}}(\widehat{\boldsymbol{\Delta}} \cdot \mathbf{p})|}
\end{array}\right)_{R X}, \\
\left.\zeta_{2}\right|_{R X} & =\left(\begin{array}{c}
0 \\
\frac{\boldsymbol{\Delta} \times \mathbf{p}}{|\boldsymbol{\Delta} \times \mathbf{p}|}
\end{array}\right)_{R X}, \\
\left.\zeta_{3}\right|_{R X} & =\frac{1}{\sqrt{-t}}\left(\begin{array}{c}
\Delta^{0} \\
\boldsymbol{\Delta}
\end{array}\right)_{R X} .
\end{aligned}
$$

The vectors $\zeta_{0}$ and $\zeta_{3}$ correspond to Pomeron helicity zero and

$$
\zeta_{ \pm}=\mp \frac{1}{\sqrt{2}}\left(\zeta_{1} \pm i \zeta_{2}\right)
$$

to helicity \pm 1 .

The expansion of $\tilde{\rho}^{\beta \alpha}$ in the helicity basis is easily obtained. We note first that

$$
p+\tilde{p}=a_{0} \zeta_{0}+a_{1} \zeta_{1},
$$

where

$$
\begin{aligned}
a_{0} & =\frac{2 \sqrt{-t}}{\xi}\left(1-\frac{1}{2} \xi\right)\left(1+\frac{4 \beta^{2} t}{Q^{2}}\right)^{-1 / 2} \\
a_{1} & =\left(a_{0}^{2}-4 m_{p}^{2}+t\right)^{1 / 2} \\
& =\frac{2}{\xi}\left[-t(1-\xi)-m_{p}^{2} \xi^{2}+\frac{\left(4 m_{p}^{2}-t\right)(-t) \beta^{2} \xi^{2}}{Q^{2}}\right]^{1 / 2}\left(1+\frac{4 \beta^{2} t}{Q^{2}}\right)^{-1 / 2}
\end{aligned}
$$

From (5.23) and (5.32)-(5.34) we get

$$
\begin{aligned}
\tilde{\rho}^{\beta \alpha}= & \frac{1}{2}\left\{\left(a_{0}^{2} \tilde{G}^{2}+t G_{M}^{2}\right) \zeta_{0}^{\beta} \zeta_{0}^{\alpha *}\right. \\
& +\frac{1}{2}\left(a_{1}^{2} \tilde{G}^{2}-2 t G_{M}^{2}\right)\left(\zeta_{+}^{\beta} \zeta_{+}^{\alpha *}+\zeta_{-}^{\beta} \zeta_{-}^{\alpha *}\right) \\
& -\frac{1}{\sqrt{2}} a_{0} a_{1} \tilde{G}^{2}\left(\zeta_{0}^{\beta} \zeta_{+}^{\alpha *}+\zeta_{+}^{\beta} \zeta_{0}^{\alpha *}\right) \\
& +\frac{1}{\sqrt{2}} a_{0} a_{1} \tilde{G}^{2}\left(\zeta_{0}^{\beta} \zeta_{-}^{\alpha *}+\zeta_{-}^{\beta} \zeta_{0}^{\alpha *}\right) \\
& \left.-\frac{1}{2} a_{1}^{2} \tilde{G}^{2}\left(\zeta_{+}^{\beta} \zeta_{-}^{\alpha *}+\zeta_{-}^{\beta} \zeta_{+}^{\alpha *}\right)\right\} \\
=: & \zeta_{s}^{\beta} \tilde{\rho}_{s r} \zeta_{r}^{\alpha *} \\
= & 9 \beta_{q}^{2}|P(\xi)|^{2} \tilde{\rho}_{s r} .
\end{aligned}
$$


Note that $\zeta_{3}=\Delta / \sqrt{-t}$ does not occur in (5.35) since the proton-Pomeron coupling is taken to be proportional to the electromagnetic coupling where the current is conserved.

We define now in accordance with (5.8):

$$
\begin{aligned}
S_{\mu r, \nu s} & =\frac{1}{2 m_{p}} \sum_{f \in X} \delta\left(\Delta+q-p_{f}\right)\left(\mathcal{M}_{\mu \alpha}^{f} \zeta_{r}^{\alpha}\right)^{*}\left(\mathcal{M}_{\nu \beta}^{f} \zeta_{s}^{\beta}\right), \\
S_{m r, n s} & =\frac{1}{2 m_{p}} \sum_{f \in X} \delta\left(\Delta+q-p_{f}\right)\left(\mathcal{M}_{\mu \alpha}^{f} \tilde{\varepsilon}_{m}^{\mu} \zeta_{r}^{\alpha}\right)^{*}\left(\mathcal{M}_{\nu \beta}^{f} \tilde{\varepsilon}_{n}^{\nu} \zeta_{s}^{\beta}\right) .
\end{aligned}
$$

From $(5.20)$ we get then:

$$
\begin{aligned}
W_{\mu \nu}^{(X)} & =S_{\mu r, \nu s} \rho_{s r}, \\
\tilde{\sigma}_{m n}^{(X)} & =\frac{4 \pi^{2} \alpha}{K} S_{m r, n s} \rho_{s r} .
\end{aligned}
$$

These are the explicit forms of $(5.6),(5.7)$ in the Donnachie-Landshoff model where the Pomeron helicities $r, s$ take the values $0, \pm$. Using (5.12) we can write $\tilde{\sigma}_{m n}^{(X)}$ as follows:

$$
\begin{aligned}
& \tilde{\sigma}_{++}^{(X)}=\frac{4 \pi^{2} \alpha}{K}\left\{S_{+0,+0} \rho_{00}+S_{++,++} \rho_{++}+S_{+-,+-} \rho_{--}\right\} \\
& \tilde{\sigma}_{00}^{(X)}=\frac{4 \pi^{2} \alpha}{K}\left\{S_{00,00} \rho_{00}+S_{0+, 0+} \rho_{++}+S_{0-, 0-} \rho_{--}\right\} \\
& \tilde{\sigma}_{+-}^{(X)}=\frac{4 \pi^{2} \alpha}{K}\left\{S_{++,--} \rho_{-+}\right\} \\
& \tilde{\sigma}_{+0}^{(X)}=\frac{4 \pi^{2} \alpha}{K}\left\{S_{++, 00} \rho_{0+}+S_{+0,0-} \rho_{-0}\right\} .
\end{aligned}
$$

Let us now discuss the behaviour of $\rho_{s r}$ for $\xi \ll 1$. We get from $(5.33)-(5.36)$ in this limit:

$$
\left(\begin{array}{c}
\rho_{00} \\
\rho_{++}=\rho_{--} \\
\rho_{0+}=\rho_{+0} \\
\rho_{0-}=\rho_{-0} \\
\rho_{+-}=\rho_{-+}
\end{array}\right) \rightarrow 9 \beta_{q}^{2}|P(\xi)|^{2} \frac{|t|}{\xi^{2}} \tilde{G}^{2}(t)\left(\begin{array}{c}
2 \\
1 \\
-\sqrt{2} \\
\sqrt{2} \\
-1
\end{array}\right)
$$

Thus all density matrix elements $\rho_{s r}$ are of the same order as $\xi \rightarrow 0$. However, the vector $\zeta_{0}$ in (5.30) contains a factor $1 / \sqrt{-t}$ whereas $\zeta_{ \pm}$(5.31) do not. Now the Pomeron is not expected to couple in general to a conserved current. Especially in inelastic diffraction there is clear evidence that it does not 99. Thus we expect

$$
\mathcal{M}_{\mu \alpha}^{f} \Delta^{\alpha}=\left.\Delta^{0}\left(\mathcal{M}_{\mu 0}^{f}-\mathcal{M}_{\mu}^{f j} \widehat{\Delta}^{j} \frac{|\Delta|}{\Delta^{0}}\right)\right|_{R X} \neq 0 .
$$


On the other hand, we can write:

$$
\mathcal{M}_{\mu \alpha}^{f} \zeta_{0}^{\alpha}=\left.\frac{\Delta^{0}}{\sqrt{-t}}\left(\mathcal{M}_{\mu 0}^{f} \frac{|\Delta|}{\Delta^{0}}-\mathcal{M}_{\mu}^{f j} \widehat{\Delta}^{j}\right)\right|_{R X} .
$$

We have for $t \rightarrow 0$

$$
\begin{aligned}
& \left.\frac{|\Delta|}{\Delta^{0}}\right|_{R X} \rightarrow 1, \\
& \sqrt{-t} \zeta_{0} \rightarrow \Delta .
\end{aligned}
$$

We conclude from (5.41)-(5.43) that $\mathcal{M}_{\mu \alpha}^{f} \zeta_{0}^{\alpha}$ will behave as $1 / \sqrt{|t|}$ for $t \rightarrow 0$ :

$$
\mathcal{M}_{\mu \alpha}^{f} \zeta_{0}^{\alpha} \rightarrow \frac{1}{\sqrt{|t|}} \mathcal{M}_{\mu \alpha}^{f} \Delta^{\alpha} .
$$

The matrix elements $\mathcal{M}_{\mu \alpha}^{f} \zeta_{ \pm}^{\alpha}$ on the other hand, have no such factor $1 / \sqrt{|t|}$ and are regular for $t=0$. If we assume that $\sqrt{|t|} \cdot \mathcal{M}_{\mu \alpha}^{f} \zeta_{0}^{\alpha} / \Delta_{R X}^{0}$ and $\mathcal{M}_{\mu \alpha}^{f} \zeta_{ \pm}^{\alpha}$ are of the same order of magnitude, we get:

$$
\begin{aligned}
S_{\mu 0, \nu 0} & \propto \frac{\left(\Delta_{R X}^{0}\right)^{2}}{|t|}, \\
S_{\mu 0, \nu \pm} & \propto \frac{\Delta_{R X}^{0}}{\sqrt{|t|}} \\
S_{\mu \pm, \nu 0} & \propto \frac{\Delta_{R X}^{0}}{\sqrt{|t|}} \\
S_{\mu \pm, \nu \pm} & \propto 1 \\
S_{\mu \pm, \nu \mp} & \propto 1 ;
\end{aligned}
$$

$$
\begin{aligned}
W_{\mu \nu}^{(X)}= & S_{\mu 0, \nu 0} \rho_{00}+ \\
& \{\text { longitudinal/transverse interference terms } \\
& \text { in Pomeron helicity of relative order } \left.\sqrt{|t|} / \Delta_{R X}^{0}\right\}+
\end{aligned}
$$$$
\{\text { transverse/transverse terms }
$$$$
\text { in Pomeron helicity of relative order } \left.|t| /\left(\Delta_{R X}^{0}\right)^{2}\right\} \text {, }
$$

where (cf. table 2):

$$
\frac{\sqrt{|t|}}{\Delta_{R X}^{0}} \simeq \frac{2 \sqrt{\beta(1-\beta)} \sqrt{|t|}}{Q}
$$


The important point in the assumption (5.45) is that we use a kinematic variable of subreaction (5.2) to compensate the $1 /|t|$ and $1 / \sqrt{|t|}$ factors in $S_{\mu 0, \nu 0}$ and $S_{\mu 0, \nu \pm}, S_{\mu \pm, \nu 0}$, respectively. Using instead of $\Delta_{R X}^{0}$ the quantities $q_{R X}^{0}$ or $Q$ or $m_{X}$ would not change the result (5.46), (5.47) in an essential way.

We see by this analysis that the Donnachie-Landshoff Pomeron couples predominantly with longitudinal helicity. This implies for the cross sections

$$
\tilde{\sigma}_{m n}^{(X)} \simeq \frac{4 \pi^{2} \alpha}{K} S_{m 0, n 0} \rho_{00}
$$

and in particular, with $(5.12)$

$$
\tilde{\sigma}_{m n}^{(X)} \simeq 0 \text { for } m \neq n .
$$

Thus we come to the following conclusions:

In the Donnachie-Landshoff Pomeron model the interference terms $\tilde{\sigma}_{m n}^{(X)}, m \neq n$, should be suppressed, since in this model the Pomeron helicity is predominantly zero. We expect from (5.39), (5.40) and (5.45)

$$
\begin{aligned}
& \frac{\tilde{\sigma}_{+0}^{(X)}}{\tilde{\sigma}_{++}^{(X)}+\tilde{\sigma}_{00}^{(X)}}=\mathcal{O}\left(\frac{2 \sqrt{\beta(1-\beta)} \sqrt{|t|}}{Q}\right), \\
& \frac{\tilde{\sigma}_{+-}^{(X)}}{\tilde{\sigma}_{++}^{(X)}+\tilde{\sigma}_{00}^{(X)}}=\mathcal{O}\left(\frac{4 \beta(1-\beta)|t|}{Q^{2}}\right) .
\end{aligned}
$$

In this way also (3.25) is satisfied for $t=-|t|_{\min }$. Indeed, we are considering here $\xi \ll 1$. Since $x \leq \xi$ we have from (A.7) $|t|_{\text {min }} \approx 0$ and from (5.50) that the interference terms $\tilde{\sigma}_{m n}^{(X)}(m \neq n)$ vanish at $t=0$. Furthermore from $(4.8) v_{X}=0$ at $t=0$ and thus from (4.11) and table 3 also the interference terms $\sigma_{m n}^{(X)}(m \neq n)$ defined in (3.17) vanish for $t=0$.

The conclusions reached above for the interference terms in the DonnachieLandshoff Pomeron model should be generically the same in other phenomenological Pomeron exchange models [26], 29]. But we must leave it to the authors of these models to give detailed predictions for the interference terms.

Let us briefly comment on the "Pomeron structure function" (cf. [1] and for a recent review [30]) as it presents itself in the light of our analysis. It is a simple exercise to show that the totally inclusive $F_{2}$ structure function of the proton can be written as follows:

$$
\begin{aligned}
F_{2}^{p}\left(x, Q^{2}\right)= & \frac{(p \cdot q)^{2}}{(p \cdot q)^{2}+p^{2} Q^{2}} \frac{Q^{2}}{2(p \cdot q)}\left(\frac{3}{2} \varepsilon_{0}^{\nu} \varepsilon_{0}^{\mu *}-\frac{1}{2} g^{\nu \mu}\right) \sum_{f}(2 \pi)^{3} \delta\left(p+q-p_{f}\right) \\
& \sum_{\text {spins }}{ }^{\prime}<f\left|J_{\mu}(0)\right| p(p)>^{*}<f\left|J_{\nu}(0)\right| p(p)>,
\end{aligned}
$$


where $\varepsilon_{0}$ is as in (3.11). For

$$
p^{2} Q^{2} \ll(p \cdot q)^{2}
$$

we get

$$
\begin{aligned}
F_{2}^{p}\left(x, Q^{2}\right) \simeq & \left(\frac{3}{2} \varepsilon_{0}^{\nu} \varepsilon_{0}^{\mu *}-\frac{1}{2} g^{\nu \mu}\right) x \sum_{f}(2 \pi)^{3} \delta\left(p+q-p_{f}\right) \\
& \sum_{\text {spins }}{ }^{\prime}<f\left|J_{\mu}(0) p(p)>^{*}<f\right| J_{\nu}(0) \mid p(p)>.
\end{aligned}
$$

For $|t| \rightarrow 0$ and with $\xi \ll 1$ and (5.52) the diffractive structure function $F_{2}^{D(4)}(3.38)$ reads in the Donnachie-Landshoff Pomeron exchange model, using (5.37), (5.38), (5.46) and (5.44),

$$
F_{2}^{D(4)}\left(x, Q^{2}, \xi, t\right)=f(\xi, t) F_{2}^{\mathbb{I P}}\left(\beta, Q^{2}\right)
$$

where $f(\xi, t)$ is the conventional "Pomeron flux factor":

$$
f(\xi, t)=\frac{9 \beta_{q}^{2}}{4 \pi^{2}}|P(\xi)|^{2} \frac{1}{\xi} \tilde{G}^{2}(t)=\frac{9 \beta_{q}^{2}}{4 \pi^{2}} \xi^{1-2 \alpha_{\mathbb{P}}(t)} \tilde{G}^{2}(t)
$$

and $F_{2}^{\mathbb{I P}}$ the "Pomeron structure function":

$$
\begin{aligned}
F_{2}^{I P}\left(\beta, Q^{2}\right) \approx & \left(\frac{3}{2} \varepsilon_{0}^{\nu} \varepsilon_{0}^{\mu *}-\frac{1}{2} g^{\nu \mu}\right) \frac{Q^{2}}{2(\Delta \cdot q)} \sum_{f \in X}(2 \pi)^{3} \delta\left(\Delta+q-p_{f}\right) \\
& \left(\mathcal{M}_{\mu \alpha}^{f} \zeta_{0}^{\alpha}\right)^{*}\left(\mathcal{M}_{\nu \beta}^{f} \zeta_{0}^{\beta}\right)|t| \\
\approx & \left(\frac{3}{2} \varepsilon_{0}^{\nu} \varepsilon_{0}^{\mu *}-\frac{1}{2} g^{\nu \mu}\right) \beta \sum_{f \in X}(2 \pi)^{3} \delta\left(\Delta+q-p_{f}\right) \\
& \left(\mathcal{M}_{\mu \alpha}^{f} \Delta^{\alpha}\right)^{*}\left(\mathcal{M}_{\nu \beta}^{f} \Delta^{\beta}\right) .
\end{aligned}
$$

Here we use the replacement (5.44) valid for small $|t|$. We see from (3.11) that $\varepsilon_{0}$ is unchanged by the substitution

$$
p \rightarrow \xi p
$$

This is obvious after setting $m_{p}^{2}=p^{2}$ in (3.11). Thus, in the approximation $\xi p \approx \Delta$, which is valid for $|t| \rightarrow 0$, the polarization vector $\varepsilon_{0}$ in (5.56) corresponding to the proton target, can be considered as the appropriate vector for the "Pomeron" target. Comparing (5.53) and (5.56) we see then that $F_{2}^{\mathbb{P}}$ has indeed the character of a Pomeron $F_{2}$ structure function since we can make the replacements

$$
\begin{aligned}
\text { proton } & \longrightarrow \text { Pomeron; } \\
p^{\mu} & \longrightarrow \Delta^{\mu}, \\
x & \longrightarrow \beta, \\
<f\left|J_{\mu}(0)\right| p(p)> & \longrightarrow \mathcal{M}_{\mu \alpha}^{f} \zeta_{0}^{\alpha} \sqrt{|t|} \approx \mathcal{M}_{\mu \alpha}^{f} \Delta^{\alpha}
\end{aligned}
$$


where we use also (5.44). However, we see that $F_{2}^{I P}$ is the "structure function" of a Pomeron with longitudinal helicity, with polarization vector $\sqrt{|t|} \zeta_{0}$. This is not a normalized polarization vector, and already from this observation we should not expect any kind of momentum sum rule for the Pomeron structure function, i.e. its parton densities, to hold.

\subsection{Soft colour interaction and "background field" models}

In this section we will first make some remarks on soft colour interaction models [15], [16]. Then we will discuss possible consequences of a picture where the QCD vacuum "background fields" play an important role [31]-34].

In [15], 16] the reaction (1.3) is viewed as proceeding in 3 steps:

(1) emission of a gluon by the original proton, leaving it as a coloured remnant $\tilde{p}_{c}$

$$
p(p) \longrightarrow G(\Delta)+\tilde{p}_{c}(\tilde{p}),
$$

(2) $\gamma^{*}-G$ fusion to give a coloured state $X_{c}$

$$
\gamma^{*}(q)+G(\Delta) \rightarrow X_{c}\left(p_{X}\right)
$$

(3) soft colour exchange where momenta are not changed:

$$
\tilde{p}_{c}(\tilde{p})+X_{c}\left(p_{X}\right) \rightarrow p(\tilde{p})+X\left(p_{X}\right) .
$$

The gluon emission in (5.59) should be governed by a vertex factor with one vector index $\alpha$, quite similar to the Pomeron emission in (5.17). Thus, the first guess would be that everything should be quite similar for the soft colour interaction models and the Pomeron exchange models. However, this is not necessarily so. To see this, we will consider replacing the gluon in (5.59) and (5.60) by a hypothetical "isoscalar photon" $\tilde{\gamma}$ which couples to a conserved current. Then (5.17) is still valid, but with $P(\xi) i 3 \beta_{q}$ replaced by a propagator factor for $\tilde{\gamma}$ :

$$
P(\xi) i 3 \beta_{q} \rightarrow P_{\tilde{\gamma}}(t)
$$

With this all the analysis from (5.19) to (5.40) remains true. However, instead of (5.41) we find now

$$
\mathcal{M}_{\mu \alpha}^{f} \Delta^{\alpha}=0
$$

We have

$$
\left.\zeta_{0}\right|_{R X}=\frac{1}{\sqrt{|t|}} \Delta+\frac{\sqrt{|t|}}{\left|\Delta_{R X}\right|+\Delta_{R X}^{0}}\left(\begin{array}{c}
1 \\
-\widehat{\Delta}
\end{array}\right)_{R X} .
$$


Together with (5.63) this leads to a suppression of longitudinal $\tilde{\gamma}$-helicity contributions for $|t| \rightarrow 0$. Instead of (5.45) we find here:

$$
\begin{aligned}
& S_{\mu 0, \nu 0} \propto \frac{|t|}{\left(\Delta_{R X}^{0}\right)^{2}}, \\
& S_{\mu 0, \nu \pm}, S_{\mu \pm, \nu 0} \propto \frac{\sqrt{|t|}}{\Delta_{R X}^{0}}, \\
& S_{\mu \pm, \nu \pm}, S_{\mu \pm, \nu \mp} \propto 1 .
\end{aligned}
$$

Thus, with exchange of the hypothetical $\tilde{\gamma}$ "photon", transverse $\tilde{\gamma}$-helicities dominate for small $|t|$ and the interference term $\tilde{\sigma}_{+-}$could be large.

We note that the discussions leading to (5.45) and (5.65), respectively, are completely analogous to the discussions for PCAC and CVC tests in inelastic neutrinonucleon scattering [35].

In the soft colour exchange models the gluon in (5.59), (5.60) should behave like our $\tilde{\gamma}$ above. However, the last step (5.61) could, a priori, change the situation and invalidate (5.63). We must again leave it to the authors of [15], [16] to give their predictions for the interference terms $\tilde{\sigma}_{m n}^{(X)}(m \neq n)$.

In the remainder of this section we will discuss the idea that the QCD "vacuum background fields" may play an important role in (1.3). We take the reaction (1.3) to proceed as in [15], 16] in the three steps (5.59)-(5.61), but in the spirit of 31 - 34 we assume them to take place in a fluctuating vacuum background field. For the hard reaction (1.3) at high $Q^{2}$ we take the vacuum fields as "frozen" (Fig. 6). It is clear that the interaction with the background field will in general invalidate angular momentum conservation arguments for the $\gamma^{*} G$ collision. The chromomagnetic Lorentz force will, for instance, lead to a correlated deflection of the coloured objects $q, \bar{q}, G$ and $\tilde{p}_{c}$. The background field may lead both to colour transfer between $X_{c}$ and $\tilde{p}_{c}$ and to angular momentum transfer. It may also lead to spin flips of $q$ and $\bar{q}$ in a way similar to the one considered for the Drell-Yan process in [32]. In such a scenario we can expect for the diffractive $\gamma^{*} p$ reaction large interference terms $\tilde{\sigma}_{m n}^{(X)}(m \neq n)$ which are in fact quantities quite analogous to the structure function $\nu$ in the Drell-Yan case (cf. [32]). Experiments for the Drell-Yan reaction $\pi^{-} N \rightarrow \gamma^{*} X$ [36] find rather large values of $\nu$ in contrast to the expectation from the QCD-improved parton model [37]. Returning to our interference terms $\tilde{\sigma}_{m n}^{(X)}$ $(m \neq n)$, we note that, of course, the boundary conditions (3.25) for $t \rightarrow-\left|t_{\text {min }}\right|$ must be respected. But guided by the Drell-Yan case we could expect here instead of (5.50) a behaviour:

$$
\frac{\tilde{\sigma}_{+0}^{(X)}}{\tilde{\sigma}_{++}^{(X)}+\tilde{\sigma}_{00}^{(X)}}=\mathcal{O}\left(\frac{\sqrt{|t|}}{M_{c}}\right),
$$




$$
\frac{\tilde{\sigma}_{+-}^{(X)}}{\tilde{\sigma}_{++}^{(X)}+\tilde{\sigma}_{00}^{(X)}}=\mathcal{O}\left(\frac{|t|}{M_{c}^{2}}\right),
$$

where $M_{c}$ is a hadronic scale, $M_{c} \simeq 1,0-1,5 \mathrm{GeV}$ say. Clearly, experiments should be able to distinguish (5.50) and (5.66). Especially in inelastic diffraction, where the proton breaks up and where the $|t|$-dependences of the cross sections are expected to be much less steep than in elastic diffraction, measurements over a large $t$-range may be feasible.

\section{Odderon contributions in $\gamma^{*} p \rightarrow \tilde{p} X$}

Let us finally make some remarks on possible "Odderon" contributions in the reaction (1.3). The Odderon, the $C=-1$ partner of the Pomeron, was discussed in the framework of Regge theory in [38]. In the framework of soft reactions in QCD developed in [12], [13] there should be an Odderon. In essence, the argument is as follows. There is no small coupling constant in the soft region. Thus, the exchange of two nonperturbative gluons between quarks, which gives the simplest contribution to the Pomeron, should be comparable to the exchange of three nonperturbative gluons which can lead to an Odderon contribution [39]. In perturbation theory, again one finds Odderon contributions [40]. Phenomenology of soft hadronic reactions, especially $p p$ and $p \bar{p}$ scattering finds, on the other hand, no significant contribution from the Odderon at small $|t|$. A possible solution to this puzzle is proposed in 41]: The Odderon coupling to quarks is intrinsically large, but its coupling to protons is suppressed. This happens if the proton has a quark-diquark structure.

In our present article we just want to point out again the possibility of looking for Odderon contributions in diffractive reactions initiated by a virtual photon (cf. [43]). Ideal reactions are ones where $X$ in (1.3) is an exclusive state with charge conjugation quantum number $C=+1$.

$$
\gamma^{*}+p \rightarrow \tilde{p}+X_{\text {excl. }}(C=+1)
$$

with, for instance,

$$
X_{\text {excl. }}=\pi^{0}, \eta, \eta^{\prime}, \eta_{c}, f_{2}(1270) .
$$

Estimates for pseudoscalar production were given in [43]. Here we will only make three more remarks:

The Odderon exchange for a reaction (6.1) where the virtual photon $\gamma^{*}$ has high $Q^{2}$ may be quite strong, even if in soft hadronic reactions the Odderon is practically absent.

If the Odderon suppression is due to the structure of the proton wave function as advocated in [41] we can expect that there is no longer such a suppression for inelastic diffraction, i.e. for the proton remnant $\tilde{p}$ different from a single proton. 
In the scenario of QCD vacuum background fields, discussed at the end of section 5.3, we should expect large Odderon contributions and thus large cross sections for the reactions (6.1), (6.2). Suppose, for instance, that the background field easily makes spin flips of $q$ and $\bar{q}$ in Fig. 6, such that their spin orientation is random after some time. Then we should expect from the number of available spin states:

$$
\frac{\sigma\left(\gamma^{*} p \rightarrow \tilde{p} \pi^{0}\right)}{\sigma\left(\gamma^{*} p \rightarrow \tilde{p} \rho^{0}\right)} \simeq \frac{1}{3}
$$

If, on the other hand, a chromomagnetic Sokolov-Ternov effect, i.e. a build-up of transverse polarizations of $q$ and $\bar{q}$ as they travel through the chromomagnetic vacuum fields (cf. [31]-[34]) is at work, we can expect to find the $q \bar{q}$ pair with oppositely oriented spins which would lead us even to the expectation

$$
\frac{\sigma\left(\gamma^{*} p \rightarrow \tilde{p} \pi^{0}\right)}{\sigma\left(\gamma^{*} p \rightarrow \tilde{p} \rho^{0}\right)} \simeq 1
$$

Similarly we could expect

$$
\frac{\sigma\left(\gamma^{*} p \rightarrow \tilde{p} \eta\right)}{\sigma\left(\gamma^{*} p \rightarrow \tilde{p} \rho^{0}\right)} \simeq \frac{\cos ^{2} \vartheta}{9} \text { or } \frac{\cos ^{2} \vartheta}{3}
$$

for the above two scenarios. Here $\vartheta \simeq-20^{\circ}$ is the flavour octet-singlet mixing angle for the pseudoscalar mesons [42].

We arrive at (6.3)-(6.5) as follows. In the constituent quark picture the flavour wave functions for $\pi^{0}, \rho^{0}$ and $\eta$ are

$$
\begin{aligned}
\pi^{0} & \sim \frac{1}{\sqrt{2}}(u \bar{u}-d \bar{d}) \\
\rho^{0} & \sim \frac{1}{\sqrt{2}}(u \bar{u}-d \bar{d}) \\
\eta & \sim \cos \vartheta \eta_{8}+\sin \vartheta \eta_{1} \\
& =\cos \vartheta \frac{1}{\sqrt{6}}(u \bar{u}+d \bar{d}-2 s \bar{s})+\sin \vartheta \frac{1}{\sqrt{3}}(u \bar{u}+d \bar{d}+s \bar{s}) \\
\eta^{\prime} & \sim-\sin \vartheta \eta_{8}+\cos \vartheta \eta_{1} .
\end{aligned}
$$

From the diagram of Fig. 6 we estimate the amplitudes for $\gamma^{*}+p \rightarrow \tilde{p}+($ meson) to be proportional to the sum of the products of the quark charges times the corresponding factor in front of $u \bar{u}, d \bar{d}$ and $s \bar{s}$, respectively, in the wave functions. In this way we get the following flavour factors for the amplitudes:

$$
\begin{aligned}
& A\left(\gamma^{*} p \rightarrow \tilde{p} \pi^{0}\right) \propto \frac{2}{3} \frac{1}{\sqrt{2}}+\frac{1}{3} \frac{1}{\sqrt{2}}=\frac{1}{\sqrt{2}}, \\
& A\left(\gamma^{*} p \rightarrow \tilde{p} \rho^{0}\right) \propto \frac{2}{3} \frac{1}{\sqrt{2}}+\frac{1}{3} \frac{1}{\sqrt{2}}=\frac{1}{\sqrt{2}}, \\
& A\left(\gamma^{*} p \rightarrow \tilde{p} \eta\right) \propto \cos \vartheta \frac{1}{\sqrt{6}}\left(\frac{2}{3}-\frac{1}{3}+\frac{2}{3}\right)+\sin \vartheta \frac{1}{\sqrt{3}}\left(\frac{2}{3}-\frac{1}{3}-\frac{1}{3}\right)=\frac{\cos \vartheta}{\sqrt{6}} .
\end{aligned}
$$


Squaring and multiplying with the spin factors $1 / 3$ or 1 we arrive at the estimates (6.3)-(6.5). In the same approximation we expect

$$
\frac{\sigma\left(\gamma^{*} p \rightarrow \tilde{p} \eta^{\prime}\right)}{\sigma\left(\gamma^{*} p \rightarrow \tilde{p} \eta\right)} \simeq \tan ^{2} \vartheta \simeq 0.13
$$

Of course, the final states in reactions (6.1), (6.2) can also be reached by the electromagnetic process where the Odderon is replaced by a virtual photon. The amplitudes for the Odderon and photon exchanges have to be added and thus there is interference which can be constructive or destructive, depending on the sign of the Odderon couplings. For further details we refer to 43], where, however, the scale on the abscissa of Fig. 3 is incorrect. An updated version of this paper is in preparation. The rate estimates given above apply to the case where the Odderon exchange dominates over photon exchange.

With this we conclude our brief discussion of "Odderon physics" in $\gamma^{*} p$ collisions.

\section{Conclusions}

In this article we have first advocated to analyse the production of rapidity gap events at HERA, i.e. the reaction $\gamma^{*} p \rightarrow \tilde{p} X$ in a way which exploits the kinematic similarity with one pion electroproduction, $\gamma^{*} p \rightarrow p \pi^{0}$. We introduced the azimuthal angle $\varphi$ between the leptonic and a hadronic plane in the rest system of the original proton and showed how $\varphi$ is related to quantities directly measurable in the HERA system. The dependence of the differential cross section on $\varphi$ was made completely explicit in sect. 3 , using the fact that the photon $\gamma^{*}$ exchanged between the lepton and the hadrons in Fig. 1 is a vector particle. We defined cross sections and interference terms $\sigma_{m n}^{(X)}$ for the absorption of photons of helicities $m, n=0,+,-$ in the proton rest frame and showed how these can be isolated using the $\varphi$ and $\varepsilon$ dependence of the cross section. The main result there is (3.34), where the $\varphi$ and $\varepsilon$ dependence is completely explicit and the $\sigma_{m n}^{(X)}$ are functions of the other kinematic variables $x, Q^{2}, \xi, t$ only. In sect. 4 the reaction $\gamma^{*} p \rightarrow \tilde{p} X$ was considered in the $X$ rest system, where we could again define cross sections and interference terms $\tilde{\sigma}_{m n}^{(X)}$. The usual structure function for diffractive events, $F_{2}^{D(4)}$ is related to $\sigma_{++}^{(X)}+\sigma_{00}^{(X)}$. The interference terms $\sigma_{m n}^{(X)}(m \neq n)$ are additional measurable quantities which can be used to test various models for diffractive events. In sect. 5 we investigated the class of models where a factorizing Pomeron is assumed to be responsible for the diffractive events. We have shown that in such models the interference terms $\tilde{\sigma}_{m n}^{(X)}(m \neq n)$ must vanish if the Pomeron is described by a density matrix which is diagonal in helicity. Thus, a nonzero $\tilde{\sigma}_{+0}^{(X)}$ and/or $\tilde{\sigma}_{+-}^{(X)}$ would rule out all models where the Pomeron has only a single helicity, for instance helicity zero. We have then analysed in detail the Donnachie-Landshoff Pomeron model. We have shown 
that there the Pomeron has predominantly helicity zero with the interference terms $\tilde{\sigma}_{+0}^{(X)}$ and $\tilde{\sigma}_{+-}^{(X)}$ being suppressed by $\sqrt{|t|} / Q$ and $|t| / Q^{2}$, respectively. We argued that in soft colour interaction models, especially if we considered possible effects of QCD vacuum background fields, the interference terms $\tilde{\sigma}_{+0}^{(X)}$ and/or $\tilde{\sigma}_{+-}^{(X)}$ could be expected to be sizable. Finally, in sect. 6, we discussed possible signals for an Odderon contribution in diffractive scattering, $\gamma^{*} p \rightarrow \tilde{p} X_{\text {excl. }}$. and speculated that such contributions could be quite large.

We hope to have given in this paper some suggestions for experimentalists what to look for in diffractive scattering at HERA in order to further elucidate the underlying dynamical mechanisms.

\section{Acknowledgements}

The authors have profited from conversations with H. Abramowicz, J. Bartels, W. Buchmüller, H. G. Dosch, F. Eisele, L. Frankfurt, G. Ingelman, H. Jung, K. Meier, H. J. Pirner, M. Rueter and S. Tapprogge. The ARC grant 313-ARC-VIII$\mathrm{VO} / \mathrm{scu}$ is gratefully acknowledged which made the mutual visits of the Cambridge and Heidelberg groups possible. M.D. and P.V.L. also acknowledge the support of PPARC and of the EU Programme "Human Capital and Mobility", Network "Physics at High Energy Colliders", Contract CHRX-CT93-0357 (DG 12 COMA), and Contract ERBCHBI-CT94-1342.

\section{Appendix A}

In this appendix we will discuss in which kinematic situations $n^{2}=0$ occurs where $n$ is defined in (2.8). For $n^{2}=0$ the angle $\varphi$ is undefined (cf. (2.10)). The discussion is best done in the $\gamma^{*}$-proton c.m. system (hcm), which is also the c.m. system of the final state hadrons. We have from (2.8)

$$
n_{h c m}=\frac{W}{m_{p}}\left(\begin{array}{c}
0 \\
\mathbf{q} \times \mathbf{p}_{X}
\end{array}\right)_{h c m} .
$$

This shows that $n^{2}$ vanishes if and only if $\mathbf{q}_{h c m}$ and $\left.\mathbf{p}_{X}\right|_{h c m}$ are collinear. This is the case for $t=-|t|_{\min }$ and $t=-|t|_{\max }$. Here $|t|_{\min }\left(|t|_{\max }\right)$ is the minimal (maximal) value of $|t|$ at fixed $W^{2}, Q^{2}, \tilde{m}^{2}, m_{X}^{2}$ which is reached for $\tilde{\mathbf{p}}_{h c m}=-\left.\mathbf{p}_{X}\right|_{h c m}$ parallel (antiparallel) to $\mathbf{p}_{h c m}=-\mathbf{q}_{h c m}$. For diffractive scattering the case $t=-|t|_{\max }$ (back scattering) is expected to correspond to very small cross section, and thus is of no practical interest. A simple calculation gives

$$
|t|_{\min }^{\min }=\left(2 p^{0} \tilde{p}^{0} \mp 2|\mathbf{p} \| \tilde{\mathbf{p}}|-m_{p}^{2}-\tilde{m}^{2}\right)_{h c m},
$$




$$
\begin{aligned}
& |t|_{\min }=\left\{\tilde{m}^{2}\left(\frac{2 p^{0}}{\tilde{p}^{0}+|\tilde{\mathbf{p}}|}-1\right)+m_{p}^{2}\left(\frac{2 \tilde{p}^{0}}{p^{0}+|\mathbf{p}|}-1\right)\right. \\
& \left.-2 \frac{m_{p}^{2} \tilde{m}^{2}}{\left(p^{0}+|\mathbf{p}|\right)\left(\tilde{p}^{0}+|\tilde{\mathbf{p}}|\right)}\right\}_{h c m}, \\
& p_{h c m}^{0}=\frac{Q^{2}}{2 W x}\left(1+\frac{2 m_{p}^{2} x}{Q^{2}}\right), \\
& |\mathbf{p}|_{h c m}=\frac{Q^{2}}{2 W x}\left(1+\frac{4 m_{p}^{2} x^{2}}{Q^{2}}\right)^{1 / 2}, \\
& \tilde{p}_{h c m}^{0}=\frac{1}{2 W} \frac{(1-x) Q^{2}-x m_{X}^{2}}{x}\left[1+\frac{x\left(m_{p}^{2}+\tilde{m}^{2}\right)}{(1-x) Q^{2}-x m_{X}^{2}}\right] \\
& |\tilde{\mathbf{p}}|_{h c m}=\frac{1}{2 W} \frac{(1-x) Q^{2}-x m_{X}^{2}}{x}\left\{1+\frac{2 x\left(m_{p}^{2}+\tilde{m}^{2}\right)}{(1-x) Q^{2}-x m_{X}^{2}}\right. \\
& \left.-\frac{4 x(1-x) Q^{2} \tilde{m}^{2}}{\left[(1-x) Q^{2}-x m_{X}^{2}\right]^{2}}+\frac{x^{2}\left(\tilde{m}^{2}-m_{p}^{2}\right)^{2}}{\left[(1-x) Q^{2}-x m_{X}^{2}\right]^{2}}\right\}^{1 / 2} .
\end{aligned}
$$

For $m_{X}^{2}$ of order $Q^{2}$ (cf. table 2) and $Q^{2} \gg m_{p}^{2}, \tilde{m}^{2}$ we obtain

$$
\begin{aligned}
|t|_{\min }= & x \frac{Q^{2}+m_{X}^{2}}{Q^{2}}\left[\tilde{m}^{2} \frac{Q^{2}}{Q^{2}-x\left(Q^{2}+m_{X}^{2}\right)}-m_{p}^{2}\right] \\
& +\mathcal{O}\left(\frac{m_{p}^{4}}{Q^{2}}, \frac{\tilde{m}^{4}}{Q^{2}}, \frac{m_{p}^{2} \tilde{m}^{2}}{Q^{2}}\right) .
\end{aligned}
$$

For the case of the proton remnant being again a single proton we get with $\tilde{m}^{2}=m_{p}^{2}$ :

$$
|t|_{\min }=x^{2} \frac{\left(Q^{2}+m_{X}^{2}\right)^{2}}{Q^{2}\left[Q^{2}-x\left(Q^{2}+m_{X}^{2}\right)\right]} m_{p}^{2}+\mathcal{O}\left(\frac{m_{p}^{4}}{Q^{2}}\right) .
$$

\section{Appendix B}

In this appendix we discuss the more general reaction $(n=1,2, \ldots ; \tilde{n}=1,2, \ldots)$

$$
\begin{aligned}
e^{\mp}(k)+p(p) \rightarrow e^{\mp}\left(k^{\prime}\right) & +a_{1}\left(p_{1}\right)+\ldots+a_{n}\left(p_{n}\right) \\
& +\tilde{a}_{1}\left(\tilde{p}_{1}\right)+\ldots+\tilde{a}_{\tilde{n}}\left(\tilde{p}_{\tilde{n}}\right),
\end{aligned}
$$

where the proton remnant is a group of particles $\tilde{a}_{1}, \ldots, \tilde{a}_{\tilde{n}}$ with momenta $\tilde{p}_{1}, \ldots, \tilde{p}_{\tilde{n}}$ and

$$
\sum_{j=1}^{\tilde{n}} \tilde{p}_{j}=\tilde{p}
$$


In order to make the dependence of the cross section for the reaction $(\mathbb{B} .1)$ on $\varphi$ again completely explicit, one can use the criteria III or IV given in sect. 2 to define the hadronic system $\tilde{p}$. When the proton remnant is no longer a single proton, one needs an additional kinematical variable, e.g. $\tilde{m}^{2} \equiv \tilde{p}^{2}$ in addition to $\mathbf{k}^{\prime}$ and $\tilde{\mathbf{p}}$ (cf. (3.7)) to describe the process (1.1) completely. Now, the cross section for the reaction (1.1) reads

$$
d \sigma\left(e^{\mp} p \rightarrow e^{\mp} \tilde{p} X\right)=\frac{4 m_{p}}{s-m_{p}^{2}}\left(\frac{\alpha}{Q^{2}}\right)^{2} l_{\nu \mu} W^{(X, \tilde{p}) \mu \nu} \frac{d^{3} k^{\prime}}{k^{\prime^{0}}} \frac{d^{3} \tilde{p}}{\tilde{p}^{0}} d \tilde{m}^{2}
$$

where

$$
\begin{aligned}
W^{(X, \tilde{p}) \mu \nu}= & \frac{1}{8 \pi m_{p}} \sum_{n} \sum_{\tilde{n}} \int_{p_{1}} \ldots \int_{p_{n}} \int_{\tilde{p}_{1}} \ldots \int_{\tilde{p}_{\tilde{n}}} \prod_{i=1}^{n} \frac{d^{3} p_{i}}{(2 \pi)^{3} 2 p_{i}^{0}} \\
& \prod_{j=1}^{\tilde{n}} \frac{d^{3} \tilde{p}_{j}}{(2 \pi)^{3} 2 \tilde{p}_{j}^{0}} \sum_{\text {spins }}{ }^{\prime}<p(p)\left|J^{\mu}(0)\right| a_{i}\left(p_{i}\right) \tilde{a}_{j}\left(\tilde{p}_{j}\right) \text { out }> \\
& <a_{i}\left(p_{i}\right) \tilde{a}_{j}\left(\tilde{p}_{j}\right) \text { out }\left|J^{\nu}(0)\right| \mathrm{p}(\mathrm{p})>\delta\left(\tilde{\mathrm{p}}-\sum_{j=1}^{\tilde{\mathrm{n}}} \tilde{\mathrm{p}}_{\mathrm{j}}\right) \\
& \chi_{n}\left(p_{1}, \ldots, p_{n}, p, q\right) \tilde{\chi}_{\tilde{n}}\left(\tilde{p}_{1}, \ldots, \tilde{p}_{\tilde{n}}, p, q\right) \\
& (2 \pi)^{4} \delta\left(p+q-\tilde{p}-\sum_{i=1}^{n} p_{i}\right) .
\end{aligned}
$$

For criterion III $\chi_{n}$ is given by $(3.22), \chi_{n}^{(I I I)}=\chi_{n}^{(I)}$ and

$$
\tilde{\chi}_{n}^{(I I I)}\left(\tilde{p}_{1}, \ldots, \tilde{p}_{\tilde{n}}, p, q\right)=\Theta\left(\tilde{m}_{c u t}^{2}-\left(\tilde{p}_{1}+\ldots+\tilde{p}_{\tilde{n}}\right)^{2}\right) .
$$

For criterion IV we have to set $\chi_{n}^{(I V)}=\chi_{n}^{(I I)}$ from $(3.23)$ and

$$
\tilde{\chi}_{n}^{(I V)}\left(\tilde{p}_{1}, \ldots, \tilde{p}_{n}, p, q\right)=\prod_{j=1}^{\tilde{n}} \Theta\left(\tilde{M}_{c u t}^{2}-\tilde{p} \cdot \tilde{p}_{j}\right) .
$$

With the definition (cf. (3.17)):

$$
\sigma_{m n}^{(X, \tilde{p})}:=\frac{4 \pi^{2} \alpha}{K} \varepsilon_{m}^{* \mu} W_{\mu \nu}^{(X, \tilde{p})} \varepsilon_{n}^{\nu}
$$

we find again that the relations (3.18), (3.19) and (3.31) hold. In analogy to (3.34) we get then

$$
\begin{aligned}
\frac{d^{7} \sigma(e p \rightarrow e \tilde{p} X)}{d x d Q^{2} d \xi d t d \varphi d \tilde{m}^{2} d \tilde{\psi}}= & \frac{1}{2 \pi} \tilde{\Gamma}\left\{\sigma_{++}^{(X, \tilde{p})}+\varepsilon \sigma_{00}^{(X, \tilde{p})}\right. \\
& -\varepsilon \cos 2 \varphi \sigma_{+-}^{(X, \tilde{p})} \\
& -2 \sqrt{\varepsilon(1+\varepsilon)} \cos \varphi \operatorname{Re} \sigma_{+0}^{(X, \tilde{p})} \\
& \left.+2 r_{L} \sqrt{\varepsilon(1-\varepsilon)} \sin \varphi \operatorname{Im} \sigma_{+0}^{(X, \tilde{p})}\right\}
\end{aligned}
$$


The cross sections and interference terms $\sigma_{m n}^{(X, \tilde{p})}$ for definite $\gamma^{*}$ helicities now depend on 5 kinematical variables

$$
\sigma_{m n}^{(X, \tilde{p})}=\sigma_{m n}^{(X, \tilde{p})}\left(x, Q^{2}, \xi, t, \tilde{m}^{2}\right) .
$$

The analyses presented in sections 4,5 , and 6 for the case of elastic diffraction $(\tilde{p}=$ single proton), are - with obvious replacements - valid also for the more general case of inelastic diffraction considered in this appendix.

\section{Appendix $\mathrm{C}$}

In (3.19) we asserted that the matrix $\left(\sigma_{m n}^{(X)}\right)$ must be positive semi-definite. The condition for this is that for every complex vector $\left(c_{m}\right)$

$$
\sum_{m, m^{\prime}} c_{m}^{*} \sigma_{m m^{\prime}}^{(X)} c_{m^{\prime}} \geq 0
$$

Indeed, inserting the definition of $\sigma_{m m^{\prime}}^{(X)}$ from (3.17), (3.9) we get

$$
\begin{aligned}
& \sum_{m, m^{\prime}} c_{m}^{*} \sigma_{m m^{\prime}}^{(X)} c_{m^{\prime}}=\frac{4 \pi^{2} \alpha}{K} \sum_{m, m^{\prime}} c_{m}^{*} \varepsilon_{m}^{* \mu} W_{\mu \nu}^{(X)} \varepsilon_{m^{\prime}}^{\nu} c_{m^{\prime}} \\
& =\frac{4 \pi^{2} \alpha}{K} \frac{1}{4 m_{p}} \sum_{n} \int_{p_{1}} \ldots \int_{p_{n}} \prod_{i=1}^{n} \frac{d^{3} p_{i}}{(2 \pi)^{3} 2 p_{i}^{0}} \\
& \chi_{n}\left(p_{1}, \ldots, p_{n}, p, q, \tilde{p}\right) \delta\left(p+q-\tilde{p}-\sum_{i=1}^{n} p_{i}\right) \\
& \sum_{\text {spins }}{ }^{\prime} \mid \sum_{m^{\prime}}<a_{i}\left(p_{i}\right) p(\tilde{p}) \text { out }\left|J_{\nu}(0)\right| p(p)>\left.\varepsilon_{m^{\prime}}^{\nu} c_{m^{\prime}}\right|^{2} \geq 0 .
\end{aligned}
$$

Under the assumption that (3.32) holds, the matrix $\left(\sigma_{m n}^{(X)}\right)$ reads

$$
\underline{\sigma}=\left(\begin{array}{ccc}
\sigma_{00}^{(X)} & \sigma_{0+}^{(X)} & -\sigma_{0+}^{(X)} \\
\sigma_{+0}^{(X)} & \sigma_{++}^{(X)} & \sigma_{+-}^{(X)} \\
-\sigma_{+0}^{(X)} & \sigma_{+-}^{(X)} & \sigma_{++}^{(X)}
\end{array}\right)
$$

with eigenvalues

$$
\begin{aligned}
\lambda_{1}= & \sigma_{++}^{(X)}+\sigma_{+-}^{(X)} \\
\lambda_{2,3}= & \frac{1}{2}\left[\sigma_{00}^{(X)}+\sigma_{++}^{(X)}-\sigma_{+-}^{(X)}\right. \\
& \left. \pm \sqrt{\left(\sigma_{00}^{(X)}+\sigma_{++}^{(X)}-\sigma_{+-}^{(X)}\right)^{2}+4\left(\sigma_{+-}^{(X)} \sigma_{00}^{(X)}+2\left|\sigma_{+0}^{(X)}\right|^{2}-\sigma_{++}^{(X)} \sigma_{00}^{(X)}\right)}\right] .
\end{aligned}
$$


The necessary and sufficient condition for the matrix $\left(\sigma_{m n}^{(X)}\right)$ to be positive semidefinite is

$$
\lambda_{i} \geq 0, \quad i=1,2,3
$$

Condition (C.5) is equivalent to

$$
\begin{aligned}
& \sigma_{00}^{(X)} \geq 0 \\
& \sigma_{++}^{(X)} \pm \sigma_{+-}^{(X)} \geq 0 \\
& \sigma_{00}^{(X)}\left(\sigma_{++}^{(X)}-\sigma_{+-}^{(X)}\right) \geq 2\left|\sigma_{+0}^{(X)}\right|^{2}
\end{aligned}
$$

These could be used in an experimental analysis in the following way. Suppose one has measured the $\varphi$-integrated cross section (cf. (3.37)):

$$
\sigma_{\varepsilon}=\sigma_{++}^{(X)}+\varepsilon \sigma_{00}^{(X)}
$$

Typically one has only a small range in $\varepsilon$ available and a separation of $\sigma_{++}^{(X)}$ and $\sigma_{00}^{(X)}$ is very difficult. Let us for simplicity assume that just one value of $\varepsilon$ is available, but that in addition to $\sigma_{\varepsilon}$ one has measured the interference terms $\sigma_{+-}^{(X)}$ and $\operatorname{Re} \sigma_{+0}^{(X)}$ (cf. (3.36) $)$. Then the allowed range for $\sigma_{00}^{(X)}$ and $\sigma_{++}^{(X)}$ in the $\sigma_{++}^{(X)}-\sigma_{00}^{(X)}$ plane can be constructed as shown in Figs. $7(\mathrm{a}, \mathrm{b})$. In particular we see that $\operatorname{Re} \sigma_{+0}^{(X)} \neq 0$ implies, of course, $\sigma_{00}^{(X)} \neq 0$.

\section{Appendix D}

Here we give the full details of the argument leading to (5.12). Consider the amplitude for $\gamma^{*}$ with helicity $n$ and $\mathbb{P}$ with helicity $s$ leading to a final state $f \in X$ (cf. 5.5$)-(5.8))$ :

$$
\mathcal{M}_{n s}^{f}=\mathcal{M}_{\mu s}^{f} \tilde{\varepsilon}_{n}^{\mu}
$$

where we always work in the system $R X$. Under a rotation $R(\chi)$ by an arbitrary angle $\chi$ around the axis $\Delta_{R X}=-\mathbf{q}_{R X}$ we have

$$
\mathcal{M}_{n s}^{f}=\mathcal{M}_{n s}^{f \chi} e^{i \chi(s-n)}
$$

where $f \chi$ denotes the rotated final state $f$. This leads to

$$
\begin{aligned}
S_{m r, n s}= & \frac{1}{2 m_{p}} \sum_{f \in X} \delta\left(\Delta+q-p_{f}\right) \mathcal{M}_{m r}^{f *} \mathcal{M}_{n s}^{f} \\
= & \frac{1}{2 m_{p}} \sum_{f \in X} \delta\left(\Delta+q-p_{f}\right) \mathcal{M}_{m r}^{f \chi *} \mathcal{M}_{n s}^{f \chi} \\
& \cdot \exp [i \chi(s-n)-i \chi(r-m)] .
\end{aligned}
$$


Now we use in an essential way that with our criteria I or II the set of states $X$ over which we sum in $(\overline{\mathrm{D} .3})$ is invariant under the rotation $R(\chi)$. Then the summation over $f$ is identical to the summation over $f \chi$ and we get from (D.3):

$$
S_{m r, n s}=S_{m r, n s} \exp [i \chi(s-n-r+m)] .
$$

Since $\chi$ is arbitrary, this implies (5.12), q.e.d. 


\section{Table Captions}

Table 1 Definitions of kinematic variables for ep scattering (1.1).

Table 2 Useful relations for the kinematic variables of ep scattering (1.1).

Table 3 The matrix $\mathcal{M}$ (4.11) relating the cross sections and interference terms $\tilde{\sigma}_{m n}^{(X)}(4.10)$ to $\sigma_{m n}^{(X)}$ (3.17) where the relations (3.32) are assumed to hold.

\section{Figure Captions}

Fig. 1 Diagram for ep scattering (1.1) in the one-photon exchange approximation $\left(q=k-k^{\prime}\right)$.

Fig. 2 The coordinate system chosen in the rest system of the original proton and the definition of the angle $\varphi$.

Fig. 3 A typical final state allowed by criterion II as rapidity gap event. The momentum configuration is drawn in the $X$ rest system where we require $p_{i}^{0} \leq M_{\text {cut }}^{2} / m_{X}$ for all $i$.

Fig. 4 The definition of the azimuthal angles $\tilde{\psi}, \psi^{\prime}$ and $\varphi^{\prime}$ in the $1-2$ plane of the HERA coordinate system, where the 3 -axis is in the direction of flight of the original proton.

Fig. 5 The reaction $\gamma^{*} p \rightarrow p X$ in the rest system of $X(R X)$. In models where this reaction proceeds via Pomeron exchange the virtual photon and the Pomeron collide head on in this system.

Fig. 6 The reactions (5.59), (5.60) taking place in a QCD vacuum background colour field, here a chromomagnetic field $\mathbf{B}_{c}$.

Fig. 7 The allowed range (thick line) for $\sigma_{++}^{(X)}$ and $\sigma_{00}^{(X)}$ if $\sigma_{\varepsilon}$ (C.7) and the interference terms $\sigma_{+-}^{(X)}$ and $\operatorname{Re} \sigma_{+0}^{(X)}$ have been measured. The case $\sigma_{+-}^{(X)} \geq 0$ corresponds to (a), the case $\sigma_{+-}^{(X)}<0$ to (b). 
Table 1

$$
\begin{aligned}
& q=k-k^{\prime}, \quad \tilde{m}^{2}=\tilde{p}^{2} \\
& \Delta=p-\tilde{p}, \quad m_{X}^{2}=p_{X}^{2} ; \\
& s=(p+k)^{2} ; \\
& W^{2}=(p+q)^{2}, \quad K=\frac{W^{2}-m_{p}^{2}}{2 m_{p}} \\
& t=(p-\tilde{p})^{2}, \\
& u=\left(p-p_{X}\right)^{2} ; \quad \nu=\frac{p \cdot q}{m_{p}} \\
& Q^{2}=-q^{2}, \quad \nu=\frac{p \cdot q}{p \cdot k} \\
& x=\frac{Q^{2}}{2 p \cdot q}, \quad y=\frac{2(1-y)-2 x y m_{p}^{2}\left(s-m_{p}^{2}\right)^{-1}}{1+(1-y)^{2}+2 x y m_{p}^{2}\left(s-m_{p}^{2}\right)^{-1}} \\
& \varepsilon= \\
& \xi=\frac{\Delta \cdot q}{p \cdot q}, \\
& \beta=\frac{Q^{2}}{2 \Delta \cdot q} .
\end{aligned}
$$




\section{Table 2}

$$
\begin{aligned}
& p+q=\tilde{p}+p_{X}, \\
& \Delta+q=p_{X}, \\
& W^{2}+t+u=m_{p}^{2}+\tilde{m}^{2}+m_{X}^{2}-Q^{2} \text {, } \\
& 2 p \cdot k=s-m_{p}^{2} \text {, } \\
& 2 p \cdot q=y\left(s-m_{p}^{2}\right) \text {, } \\
& Q^{2}=x y\left(s-m_{p}^{2}\right), \\
& 2 p_{X} \cdot q=m_{X}^{2}-t-Q^{2}=-x y\left(s-m_{p}^{2}\right)+m_{X}^{2}-t, \\
& 2 p_{X} \cdot p=y\left(s-m_{p}^{2}\right)+t+m_{p}^{2}-\tilde{m}^{2}, \\
& (p \cdot q)^{2}+Q^{2} m_{p}^{2}=m_{p}^{2}\left(\nu^{2}+Q^{2}\right) \\
& =m_{p}^{2} \nu^{2}\left[1+\frac{4 x^{2} m_{p}^{2}}{Q^{2}}\right] \\
& =\frac{1}{4} y\left(s-m_{p}^{2}\right)^{2}\left[y+\frac{4 x m_{p}^{2}}{s-m_{p}^{2}}\right] \\
& \xi=\frac{m_{X}^{2}+Q^{2}-t}{W^{2}+Q^{2}-m_{p}^{2}}, \\
& \beta=\frac{x}{\xi}, \quad 0 \leq \beta \leq 1 \text {; } \\
& \beta=\frac{Q^{2}}{m_{X}^{2}+Q^{2}-t}, \\
& m_{X}^{2}=\frac{Q^{2}(1-\beta)+t \beta}{\beta}, \\
& \Delta_{R X}^{0}=\frac{\Delta \cdot p_{X}}{m_{X}}=\frac{m_{X}^{2}+Q^{2}+t}{2 m_{X}} \\
& =\frac{Q^{2}}{2 \beta m_{X}}\left(1+\frac{2 \beta t}{Q^{2}}\right) \\
& =\frac{1}{2} Q\left[\beta(1-\beta)+\frac{\beta^{2} t}{Q^{2}}\right]^{-1 / 2}\left(1+\frac{2 \beta t}{Q^{2}}\right) \text {, } \\
& |\boldsymbol{\Delta}|_{R X}=\frac{1}{2} Q\left[\beta(1-\beta)+\frac{\beta^{2} t}{Q^{2}}\right]^{-1 / 2}\left(1+\frac{4 \beta^{2} t}{Q^{2}}\right)^{1 / 2}
\end{aligned}
$$


Table 3

\begin{tabular}{l|ccccc} 
& $\sigma_{++}^{(X)}$ & $\sigma_{00}^{(X)}$ & $\sigma_{+-}^{(X)}$ & $\operatorname{Re} \sigma_{+0}^{(X)}$ & $\operatorname{Im} \sigma_{+0}^{(X)}$ \\
\hline$\tilde{\sigma}_{++}^{(X)}$ & $\left(\gamma_{X}^{2}+1\right) / 2$ & $v_{X}^{2} \gamma_{X}^{2} / 2$ & $-v_{X}^{2} \gamma_{X}^{2} / 2$ & $-\sqrt{2} v_{X} \gamma_{X}^{2}$ & 0 \\
$\tilde{\sigma}_{00}^{(X)}$ & $v_{X}^{2} \gamma_{X}^{2}$ & $\gamma_{X}^{2}$ & $-v_{X}^{2} \gamma_{X}^{2}$ & $-2 \sqrt{2} v_{X} \gamma_{X}^{2}$ & 0 \\
$\tilde{\sigma}_{+-}^{(X)}$ & $-v_{X}^{2} \gamma_{X}^{2} / 2$ & $-v_{X}^{2} \gamma_{X}^{2} / 2$ & $\left(\gamma_{X}^{2}+1\right) / 2$ & $\sqrt{2} v_{X} \gamma_{X}^{2}$ & 0 \\
$\operatorname{Re} \tilde{\sigma}_{+0}^{(X)}$ & $v_{X} \gamma_{X}^{2} / \sqrt{2}$ & $v_{X} \gamma_{X}^{2} / \sqrt{2}$ & $-v_{X} \gamma_{X}^{2} / \sqrt{2}$ & $-\left(1+v_{X}^{2}\right) \gamma_{X}^{2}$ & 0 \\
$\operatorname{Im} \tilde{\sigma}_{+0}^{(X)}$ & 0 & 0 & 0 & 0 & -1
\end{tabular}

\section{References}

[1] G. Ingelman, P.E. Schlein: Phys. Lett. B152, 256 (1985)

[2] A. Donnachie, P.V. Landshoff: Phys. Lett. B191, 309 (1987)

[3] A. Donnachie, P. V. Landshoff: Nucl. Phys. B303, 634 (1988)

[4] G. Ingelman, K. Janson-Prytz: "The Pomeron Structure Function and QCD at small $x$ ", in: Physics at HERA, eds. W. Buchmüller, G. Ingelman (DESY, Hamburg 1992), p. 233

[5] G. Ingelman, K. Prytz, Z. Phys. C58, 285 (1993)

[6] M. Derrick et al., (ZEUS coll.): Phys. Lett. B315, 481 (1993); Z. Phys. C68, 569 (1995)

[7] T. Ahmed et al., (H1 coll.): Nucl. Phys. B429, 477 (1994); Phys. Lett. B348, 681 (1995)

[8] P. D. B. Collins: "An Introduction to Regge Theory", Cambridge University Press, Cambridge, U.K. 1977;

L. Caneschi, ed: "Regge Theory of low $p_{T}$ hadronic interactions", North Holland, Amsterdam 1989

[9] A. Donnachie, P. V. Landshoff: Nucl. Phys. B244, 322 (1984); Nucl. Phys. B267, 690 (1986); Phys. Lett. B185, 403 (1987)

[10] F. E. Low: Phys. Rev. D12, 163 (1975);

S. Nussinov: Phys. Rev. Lett. 34, 1286 (1975);

J. F. Gunion, D. E. Soper: Phys. Rev. D15, 2617 (1977) 
[11] E. A. Kuraev, L. N. Lipatov, V. S. Fadin: Sov. Phys. J.E.T.P. 44, 443 (1976), 45, 199 (1977);

L. N. Lipatov: Sov. J. Nucl. Phys. 23, 338 (1976);

Ya. Ya. Balitskii, L. N. Lipatov: Sov. J. Nucl. Phys. 28, 822 (1978);

L. N. Lipatov: Sov. Phys. J.E.T.P. 63, 904 (1986);

L. N. Lipatov: "Pomeron in Quantum Chromodynamics", in: Perturbative Quantum Chromodynamics, ed. A. H. Mueller, World Scientific, Singapore, 1989;

A. R. White: Int. J. Mod. Phys. A6, 1859 (1990);

J. Bartels: Z. Phys. C60, 471 (1993)

[12] P. V. Landshoff, O. Nachtmann: Z. Phys. C35, 405 (1987)

[13] O. Nachtmann: Ann. Phys. 209, 436 (1991)

[14] H. G. Dosch, E. Ferreira, A. Krämer: Phys. Lett. B289, 153 (1992): Phys. Rev. D50, 1992 (1994)

[15] W. Buchmüller, A. Hebecker: Phys. Lett. B355, 573 (1995)

[16] A. Edin, G. Ingelman, J. Rathsman: Phys. Lett. B366, 371 (1996)

[17] W. Buchmüller, A. Hebecker: "Semiclassical Approach to Structure Functions at Small $x "$, DESY-95-208, hep-ph/9512329 (1995)

[18] O. Nachtmann: "Elementary Particle Physics", Springer Berlin, Heidelberg 1990

[19] M. Gourdin: Il Nuovo Cimento 21, 1094 (1961);

C. W. Akerlof et al., Phys. Rev. Lett. 14, 1036 (1965)

[20] N. Dombey: "Introduction to photoproduction and electroproduction" in: Hadronic Interactions of Electrons and Photons, eds. J. Cumming, H. Osborn, Academic Press, London 1971

[21] K. Berkelman: "Continuum Electroproduction by Coincidence Measurements", in: Proc. 1971 Int. Symp. on Electron and Photon Interactions at High Energies, ed. N. B. Mistry, Cornell Univ. 1972

[22] H. Georgi, H.D. Politzer: Phys. Rev. Lett. 40, 3 (1978);

G. Köpp, R. Maciejko, P.M. Zerwas: Nucl. Phys. B144, 123 (1978);

A. Méndez, A. Raychaudhuri, V.J. Stenger: Nucl. Phys. B148, 499 (1979);

R.N. Cahn: Phys. Lett. B78, 269 (1978); Phys. Rev. D40, 3107 (1989);

E.L. Berger: Phys. Lett. B89, 241 (1980); 
V. Hedberg, G. Ingelman, C. Jacobsson, L. Jönsson: "Asymmetries in jet azimuthal angle distributions as a test of QCD", in: Physics at HERA, eds. W. Buchmüller, G. Ingelman (DESY, Hamburg 1992), p. 331;

J. Chay, S.D. Ellis, W.J. Stirling: Phys. Rev. D45, 46 (1992);

R. Meng, F. I. Olness, D. E. Soper: Nucl. Phys. B371, 79 (1992)

[23] J. Ashman et al. (EMC): Z. Phys. C52, 1 (1991)

[24] Ch. Busch: "Messung hadronischer Strukturfunktionen mit dem H1 Detektor", Diploma Thesis, Univ. Heidelberg (1995), unpublished

[25] L. Hand: Phys. Rev. 129, 1834 (1964)

[26] T. Gehrmann, W. J. Stirling: Durham preprint DTP/95/26, hep-ph/9503351 (1995)

[27] L. Van Hove: Phys. Lett. 24B, 183 (1967)

[28] G. Höhler: Landolt-Börnstein, Vol. I/9b2, p. 301, ed. H. Schopper;

F. Borkowski et al.: Nucl. Phys. A222, 269 (1974); Nucl. Phys. B93, 461 (1975);

S. Platchkov et al.: Nucl. Phys. A510, 740 (1990);

P.E. Bosted et al.: Phys. Rev. Lett. 68, 3841 (1992)

[29] B. A. Kniehl, H.-G. Kohrs, G. Kramer: Z. Phys. C65, 657 (1995);

K. Golec-Biernat, J. Kwieciński: Phys. Lett. B353, 329 (1995)

[30] P. V. Landshoff: "Introduction to the Pomeron Structure Function", hepph/9505254 (1995), unpublished

[31] O. Nachtmann, A. Reiter: Z. Phys. C24, 283 (1984)

[32] A. Brandenburg, E. Mirkes, O. Nachtmann: Z. Phys. C60, 697 (1993)

[33] G. W. Botz, P. Haberl, O. Nachtmann: Z. Phys. C67, 143 (1995)

[34] O. Nachtmann: "The QCD vacuum structure and its manifestations", in: Proc. of the First ELFE Summer School, 1995, Cambridge, U.K., to be published

[35] S. L. Adler: Phys. Rev. 135, B963 (1964)

[36] S. Falciano et al. (NA10 coll.): Z. Phys. C31, 513 (1986);

M. Guanziroli et al. (NA10 coll.): Z. Phys. C37, 545 (1988)

[37] P. Chiapetta, M. LeBellac: Z. Phys. C32, 521 (1986) 
[38] L. Lukaszuk, B. Nicolescu: Nouvo Cimento Lett. 8, 405 (1973);

D. Bernard, P. Gauron, B. Nicolescu: Phys. Lett. B199, 125 (1987);

E. Leader: Phys. Lett. B253, 457 (1991)

[39] A. Donnachie, P.V. Landshoff: Nucl. Phys. B348, 297 (1991);

A. Schäfer, L. Mankiewicz, O. Nachtmann: Phys. Lett. B272, 419 (1991)

[40] L. N. Lipatov: Phys. Lett. B251, 284 (1990)

[41] M. Rueter, H. G. Dosch: "Nucleon Structure and High Energy Scattering", Univ. of Heidelberg report HD-THEP-96-04, hep-ph/9603214 (1996)

[42] Particle Data Group: Phys. Rev. D50, Vol. 3-I (1994), p. 1321

[43] A. Schäfer, L. Mankiewicz, O. Nachtmann: "Diffractive $\eta_{c}, \eta, \eta^{\prime}, J / \psi$ and $\psi^{\prime}$ production in electron-proton collisions at HERA energies", in: Physics at HERA, eds. W. Buchmüller, G. Ingelman, (DESY, Hamburg 1992), p. 243 


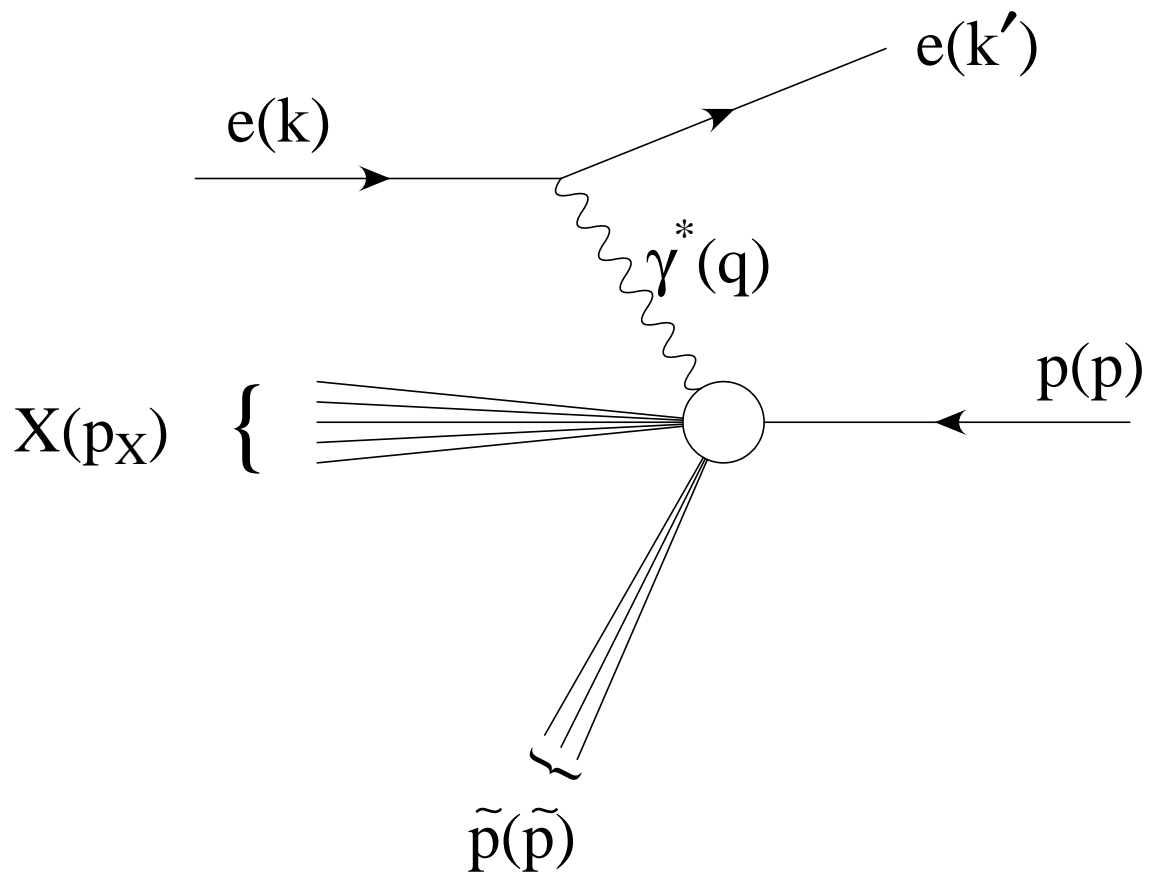

Figure 1 


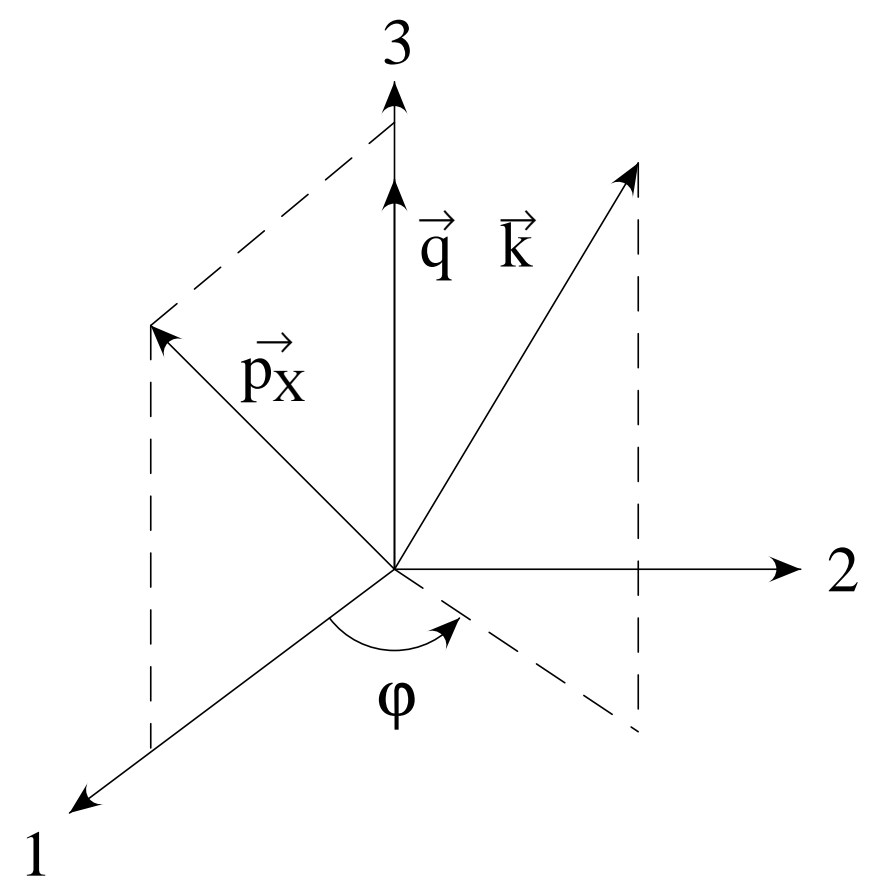

Figure 2 


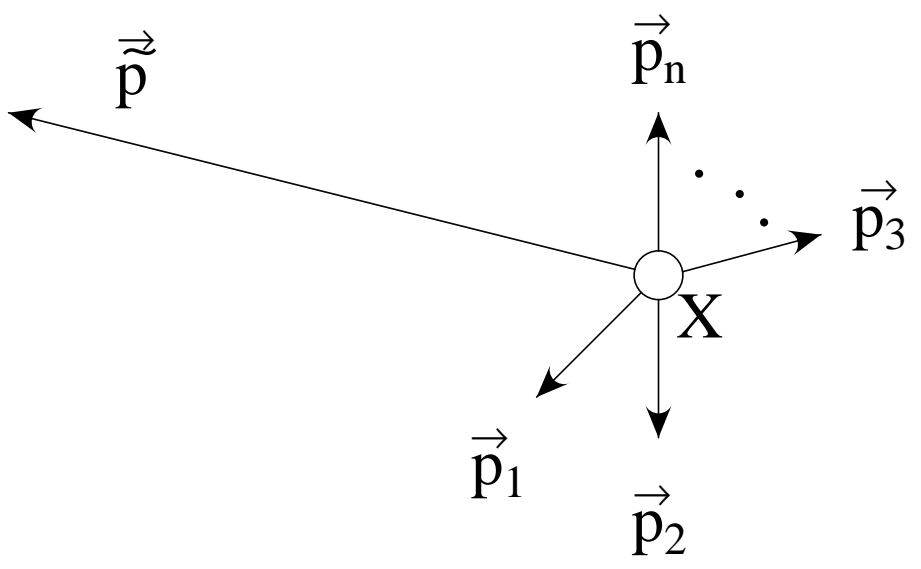

Figure 3 


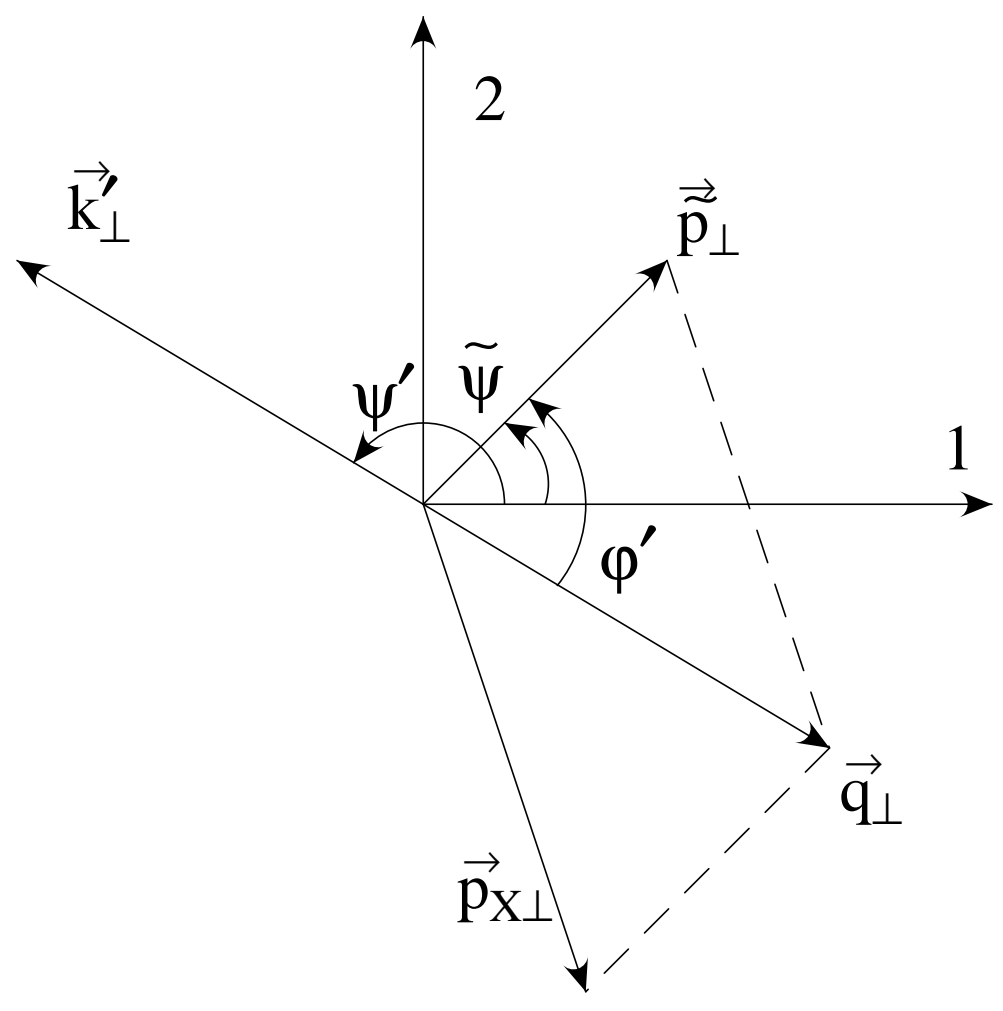

Figure 4 


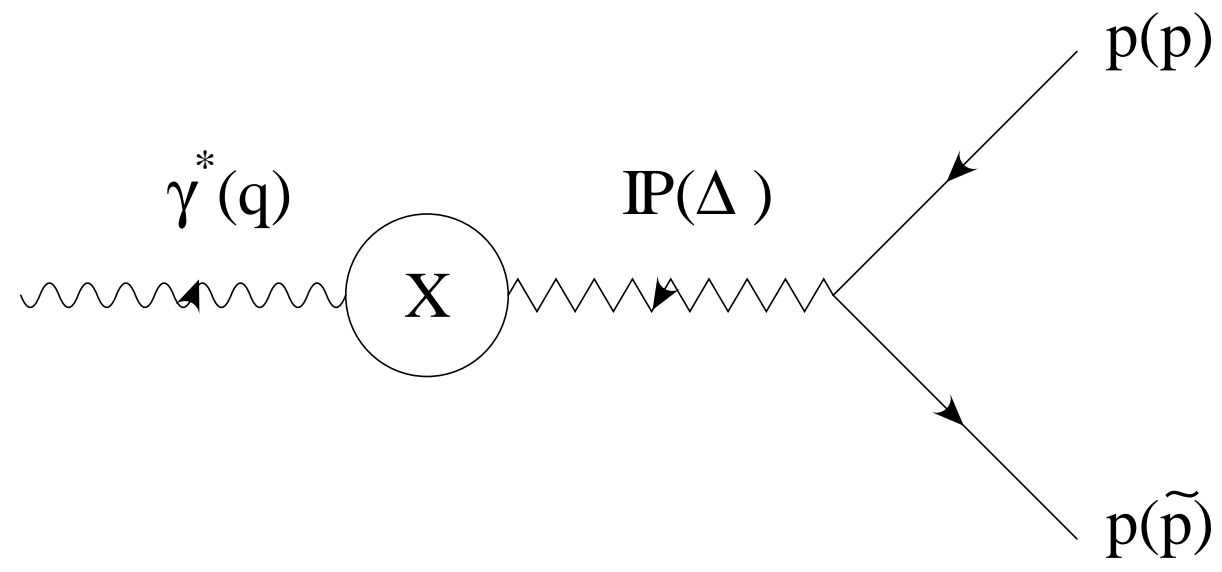

Figure 5 


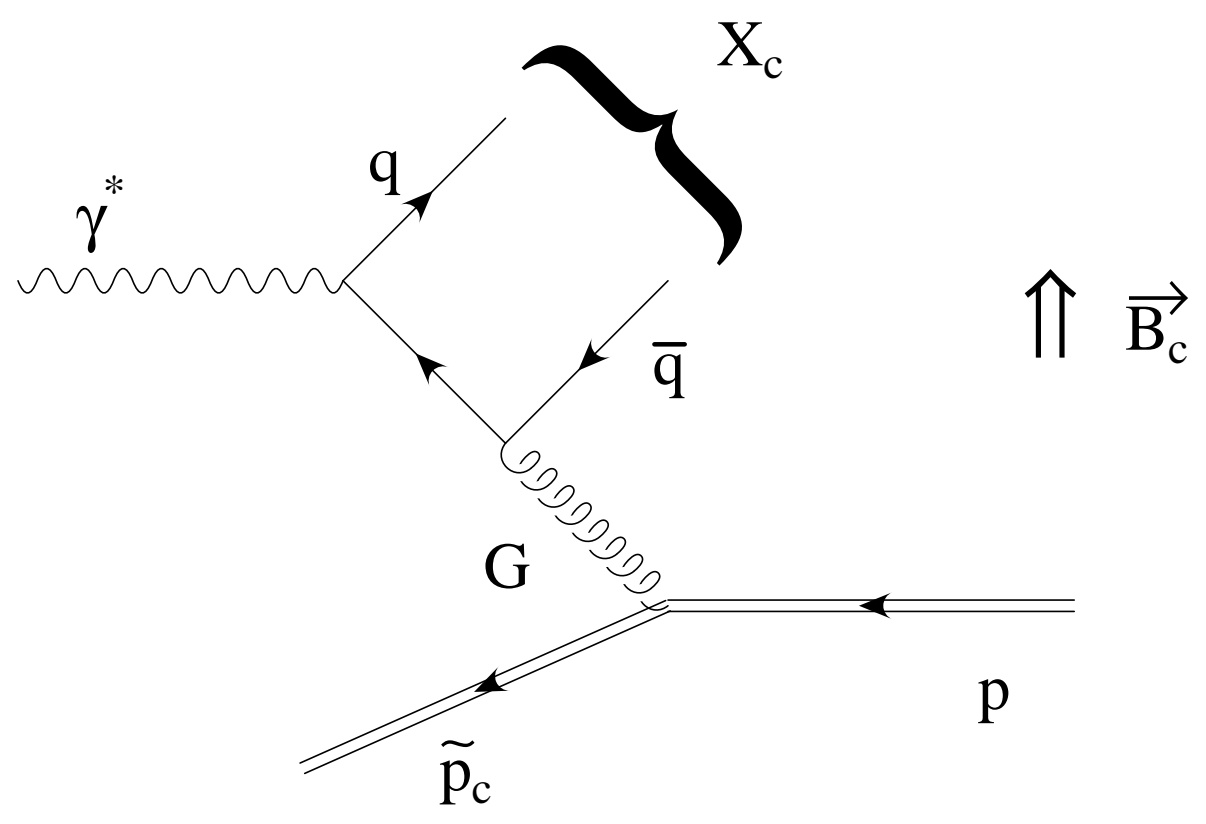

Figure 6 


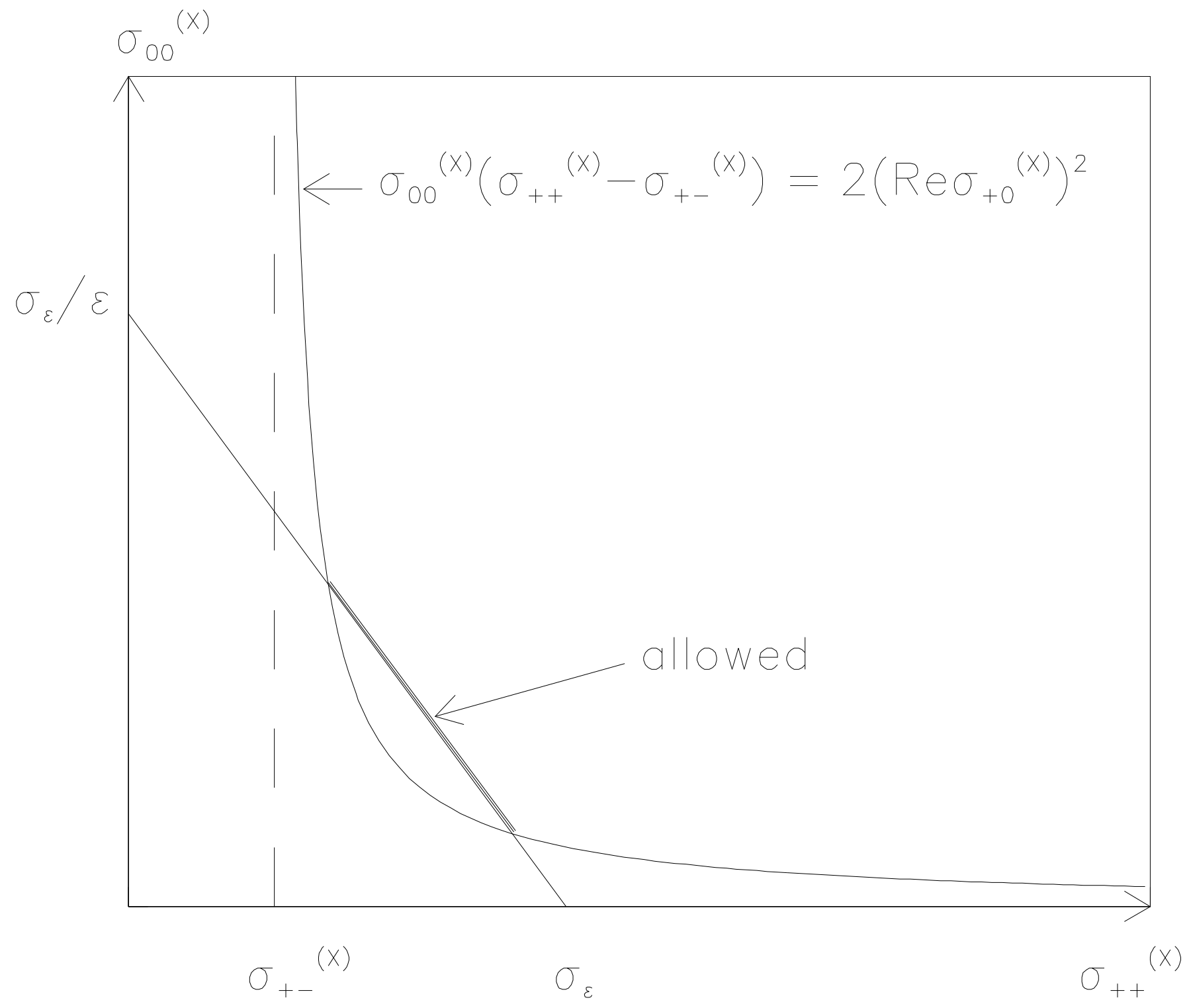

Fig. 7(a) 


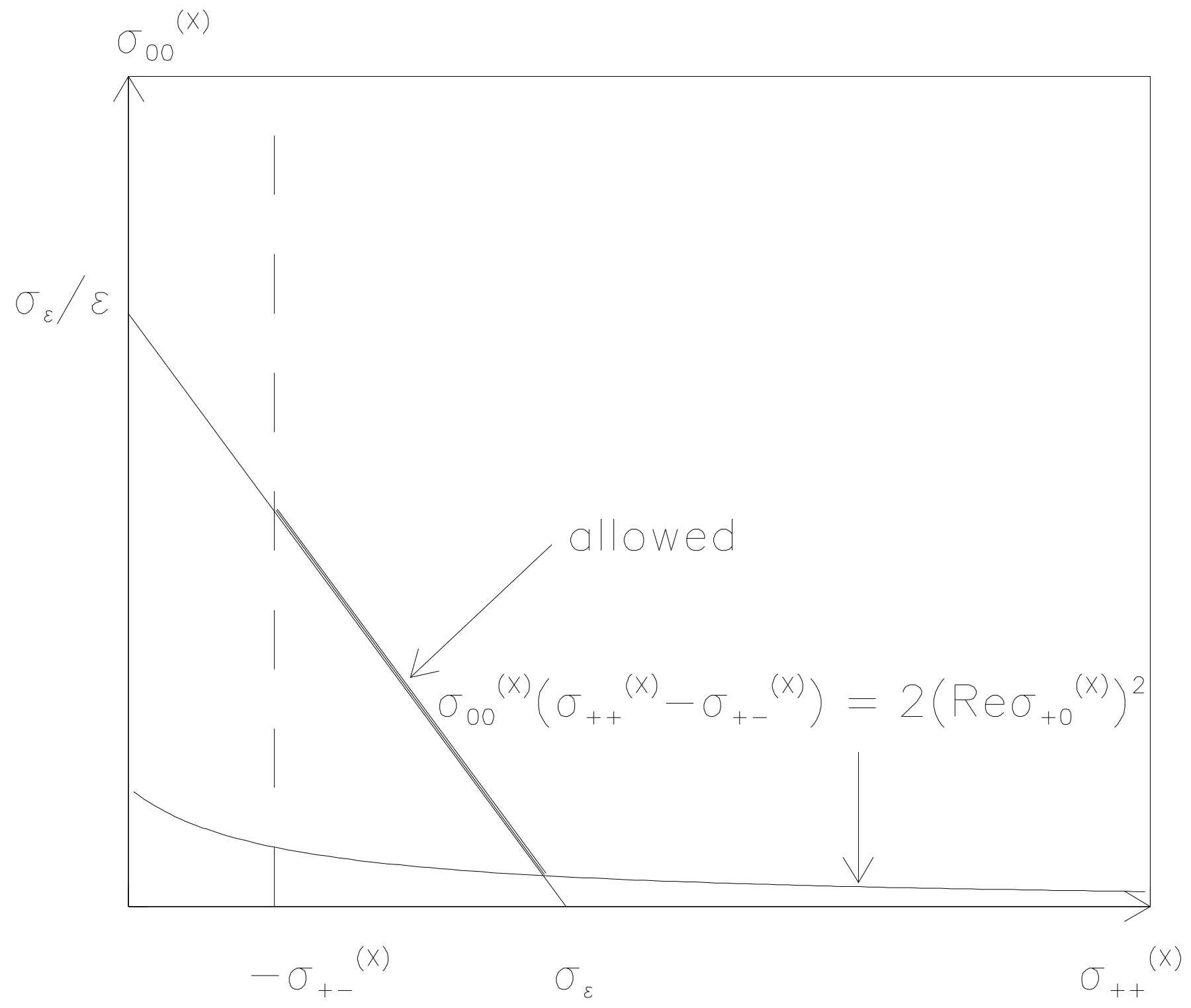

Fig. 7(b) 